\title{
LISTADO FLORÍSTICO DE LA FAMILIA POACEAE DEL ESTADO DE MORELOS, MÉXICO
}

\author{
J. Gabriel Sánchez-Ken ${ }^{1,3}$ y Rosa Cerros-Tlatilpa ${ }^{2}$ \\ ${ }^{1}$ Investigador independiente, México, D.F., México. \\ ${ }^{2}$ Universidad Autónoma del Estado de Morelos, Facultad de \\ Ciencias Biológicas, México. \\ ${ }^{3}$ Autor para la correspondencia: gabriels@hotmail.co.uk
}

\begin{abstract}
RESUMEN
Se realizó una revisión de algunos herbarios nacionales y extranjeros para la obtención del listado florístico de la familia Poaceae para el estado de Morelos, México. La familia está representada por 104 géneros, 306 especies y 10 categorías infraespecíficas, perteneciendo a ocho subfamilias siendo las Chloridoideae, Panicoideae y Pooideae, las más ricas en especies. El estado se reubica del $20^{\circ}$ al $13^{\circ}$ estado más diverso para la familia a nivel nacional. Se realizó un breve análisis del estado de conocimiento de la familia en Morelos, por municipios, así como el esfuerzo de colecta que se ha realizado a través de los años. Se registran siete especies de malezas nocivas listadas en la normatividad mexicana.
\end{abstract}

Palabras clave: diversidad, malezas nocivas, México, Morelos, Poaceae.

\begin{abstract}
Several national and foreign herbaria were reviewed to obtain the checklist of the family Poaceae of the state of Morelos, Mexico. The family is represented by 104 genera, 306 species and 10 infraspecific categories, classified in eight subfamilies with Chloridoideae, Panicoideae and Pooideae the most diverse ones. Morelos is repositioned from the $20^{\text {th }}$ to the $13^{\text {th }}$ most diverse state in Mexico with respect to species richness of the family. We present a brief analysis of the knowledge of the flora in this state by municipalities, as well as the collection effort that has been made throughout the years. Seven noxious weed species, which are listed in Mexican laws are recorded in the state.
\end{abstract}

Key words: diversity, Mexico, noxious weeds, Morelos, Poaceae. 


\section{INTRODUCCIÓN}

En los últimos años se ha incrementado el estudio de la diversidad florística de la familia Poaceae en el estado de Morelos a través de algunas publicaciones (Bárcenas, 1977; Soria, 1985; Cerros-Tlatilpa y Espejo-Serna, 1998). El trabajo de Vázquez (1974) es el primero en generar un listado de 3000 especies de plantas vasculares. De ésta lista, la familia Poaceae está representada por 65 géneros y 155 especies (Vázquez, 1974). Posteriormente, Bonilla-Barbosa y Villaseñor (2003) publicaron el Catálogo de la Flora del estado de Morelos, el cual fue una revisión bibliográfica completada con el estudio de especímenes de los herbarios HUMO y MEXU. En este catálogo se registraron 3686 especies de plantas vasculares conocidas, incluyendo especies nativas e introducidas.

Bonilla-Barbosa y Villaseñor (2003) mencionaron que la familia Poaceae con 216 especies está ubicada en el tercer lugar de diversidad florística con respecto a los demás estados de la República Mexicana. Además, señalaron la existencia de un número reducido de especies endémicas pero no mencionaron ninguna en particular. Contreras-MacBeath et al. (2004) retomaron éstos datos para la publicación de "La diversidad biológica de Morelos: estudio del estado", sin modificar ningún dato.

En el Catálogo de las Gramíneas de México, Dávila et al. (2006) ubicaron al estado de Morelos con 226 especies en el $20^{\circ}$ lugar de diversidad de gramíneas nativas e introducidas en el país. También mencionaron que el estado no tenía ninguna especie endémica exclusiva, sino más bien algunas especies con distribución restringida y compartida con los estados circundantes.

En el estado de Morelos existen varias áreas naturales protegidas de tipo federal, una de las cuales es la Reserva de la Biósfera Sierra de Huautla (REBIOSH) que fue estudiada por Dorado et al. (2005), quienes registraron 31 géneros y 66 especies de la familia Poaceae. En otra área natural protegida, el Corredor biológico del Chichinautzin, Pulido-Esparza et al. (2009) estudiaron las monocotiledóneas mencionando 38 géneros y 75 especies de gramíneas nativas. Para la misma zona un estudio reciente de Flores-Castorena y Martínez-Alvarado (2011) registró 55 géneros, 124 especies y 5 categorías infraespecíficas.

Estudios en particular sobre la diversidad florística de la familia Poaceae del estado son escasos y regionales, tal es el caso de las gramíneas de las Lagunas de Zempoala (Bonilla-Barbosa y Viana, 1997), del Tepozteco de Itie (1935), las Panicoideae y Chloridoideae del estado de Morelos (Sotelo, 1984; Bustos, 2008), las gramíneas forrajeras de Tepoztlán (Díaz-Pulido, 1998), las gramíneas del municipio de Amacuzac (Galindo-Becerril y Fernández-Nava, 2002) y recientemente las gramí- 
neas de los municipios de Amacuzac, Axochiapan, Ocuituco, Yautepec y Zacatepec por Díaz-Pulido et al. (2007a, 2007b, 2007c, 2007d, 2007e) con ciertas debilidades ya que algunas especies tienen una distribución al norte del país.

Dado el pobre conocimiento y las inconsistencias encontradas en el estudio de la familia Poaceae en el estado de Morelos, se planteó realizar una revisión más precisa y crear un listado de la familia. Una vez desarrollado este listado, el objetivo principal fue verificar que todas las especies tuvieran al menos un ejemplar de herbario, para así presentar un listado actual de la familia Poaceae para el estado de Morelos.

\section{ÁREA DE ESTUDIO}

El estado de Morelos está situado en la parte centro sur de México entre $19^{\circ} 08^{\prime}$ latitud norte a $18^{\circ} 19^{\prime}$ latitud sur y entre $98^{\circ} 38^{\prime}$ longitud este a $99^{\circ} 20^{\prime}$ longitud oeste, con una superficie de $4892.7 \mathrm{~km}^{2}$ que representa $0.25 \%$ del territorio total del país. Morelos ocupa la $30^{a}$ posición en extensión geográfica en relación con el resto de los estados de la república mexicana (INEGI, 2013).

El estado de Morelos forma parte de dos provincias fisiográficas, la Depresión del Balsas y el Eje Neovolcánico (Rzedowski, 1978). En la porción norte del estado se encuentra el Eje neovolcánico, de donde parten las serranías de Zempoala, Tres Marías, Tepoztlán, Tlayacapan y Tlalnepantla. Al noreste se encuentra la Sierra Nevada, parte del Volcán Popocatépetl, en la cual se encuentran las serranías de Chalma y Ocuilan, que se continúan con las montañas de Guerrero. Los tres ríos más importantes en cuanto a afluencia son el río Grande, el río Tepalcingo y el río Amacuzac, mientras que existen tres complejos de lagunas, El Rodeo, Coatetelco y Tequesquitengo (Rzedowski, 1978; INEGI, 2013).

Respecto a la altitud, el estado presenta áreas desde los 720 a los $5432 \mathrm{~m}$ s.n.m. Esta variación altitudinal se ve reflejada en un gradiente climático, desde zonas frías a zonas tropicales semisecas. En la mayor parte de la superficie del estado (68.1\%) predomina un clima cálido subhúmedo, en el resto uno semicálido subhúmedo (18.8\%), templado subhúmedo $(9.7 \%)$ y en $3.4 \%$ de la superficie otros tipos de clima. Dependiendo de la variación altitudinal, las temperaturas mínimas son de $12.2{ }^{\circ} \mathrm{C}$, media anual de $21.5^{\circ} \mathrm{C}$ y máximas de $32{ }^{\circ} \mathrm{C}$. El régimen de lluvias es de verano, con un promedio aproximado de $900 \mathrm{~mm}$ anuales (INEGI, 2013).

En el estado de Morelos se desarrollan ocho tipos de vegetación: bosque de coníferas, bosque de Quercus, bosque mesófilo de montaña, bosque tropical caduci- 
folio, bosque de galería, matorral rosétofilo-crassicaule, pastizal y vegetación acuática. En el norte del estado se ubican los bosques de coníferas y el pastizal alpino y hacia el sur el bosque tropical caducifolio, siendo éste último tipo el que ocupa la mayor extensión del estado (Rzedowski, 1978).

\section{MÉTODO}

Se realizó una revisión de la literatura (Apéndice 1) hasta completar una lista con los nombres de las especies para el estado de Morelos. Esta lista se depuró y se verificó la existencia de especímenes botánicos principalmente en los herbarios HUMO, MEXU, UAMIZ, UAMX, además de consultar las bases de datos o herbarios virtuales de ARIZ, B, BM, F, GH, IBUG, IEB, ISC, MICH, MO, NY, SI, TAES, US, WIS y XAL. Algunas especies sin especímenes botánicos existentes, pero que se han observado en el campo, fueron incluidas con base a referencias bibliográficas.

La clasificación de la familia y los nombres taxonómicos fueron actualizados de acuerdo al Catalogue of New World Grasses (CNWG) tanto impreso como en línea www.tropicos.org/Project/CNWG (Soreng et al., 2003; 2015). Los nombres de los autores son de acuerdo al International Plant Names Index (www.ipni.org) y autores de nombres de plantas (Brummitt y Powell, 1992).

Se desarrolló una base de datos la cual incluyó los datos de las etiquetas y los herbarios donde se encuentran depositados. También se incluyeron campos tales como usos (alimento, cereales, control de erosión, forraje, maleza (nociva o potencial), medicinal, ornamental), origen, y grupos taxonómicos al que pertenecen las especies.

Para observar como ha sido el comportamiento histórico de la recolección de especímenes, se utilizaron los años de colecta y para observar con que intensidad se han estudiado los municipios del estado, se utilizaron los números de colecta.

\section{RESULTADOS Y DISCUSIÓN}

Hasta el momento se tienen registrados en la base de datos 2048 especímenes, incluyendo duplicados de diferentes herbarios, principalmente de HUMO, MEXU, MO, UAMIZ, UAMX y US (Cuadro 1). Estos últimos seis herbarios son los que contienen el mayor número de especímenes, siendo MEXU, HUMO y US los más importantes. 
Cuadro 1. Número de especímenes incluyendo duplicados depositados en cada uno de los herbarios.

\begin{tabular}{cccc}
\hline Herbario & Especímenes & Herbario & Especímenes \\
\hline ARIZ & 3 & MICH & 2 \\
B & 1 & MO & 165 \\
BM & 1 & NY & 2 \\
F & 4 & SI & 2 \\
GH & 1 & TAES & 1 \\
HUMO & 572 & UAMIZ & 138 \\
IBUG & 8 & UAMX & 525 \\
IEB & 2 & US & 307 \\
ISC & 1 & WIS & 2 \\
MEXU & 675 & XAL & 4 \\
\hline
\end{tabular}

El listado florístico de la familia Poaceae en el Estado de Morelos consiste en 104 géneros y 306 especies, con 10 categorías infraespecíficas sin ninguna especie endémica (ver Apéndice 2).

Todas las especies, excepto dos, están debidamente respaldadas con al menos un espécimen de herbario. Las dos especies sin ejemplares de herbario representativos son Arundo donax L. y Cymbopogon citratus (DC.) Stapf, las cuales han sido observadas en campo pero no colectadas (González-Barrales, 2006; March-Mifsut y Martínez-Jiménez, 2007).

El espécimen más antiguo registrado en la base de datos fue colectado en el estado de Morelos en 1865, se encuentra depositado en el herbario MO. Sin embargo, es muy probable que existan colecciones más antiguas depositadas en herbarios europeos. La gráfica de la Figura 1 muestra la intensidad de recolección de especímenes de herbario a través de los años desde 1865 a la actualidad. El mayor número de colectas se realizó en los últimos 30 años. La Figura 2 muestra la intensidad de colecta de especímenes entre los meses de agosto a noviembre, que corresponde a la época de mayor floración de las gramíneas en todo el estado.

El análisis de la base de datos permite observar cuales son los municipios mejor colectados. El Cuadro 2 muestra los municipios de Cuernavaca, Huitzilac, Tepoztlán, Tlayacapan y Yautepec, que en conjunto concentran $18.24 \%$ de la extensión geográfica del estado y son los mejor estudiados con $46 \%$ del total de especímenes registrados. 


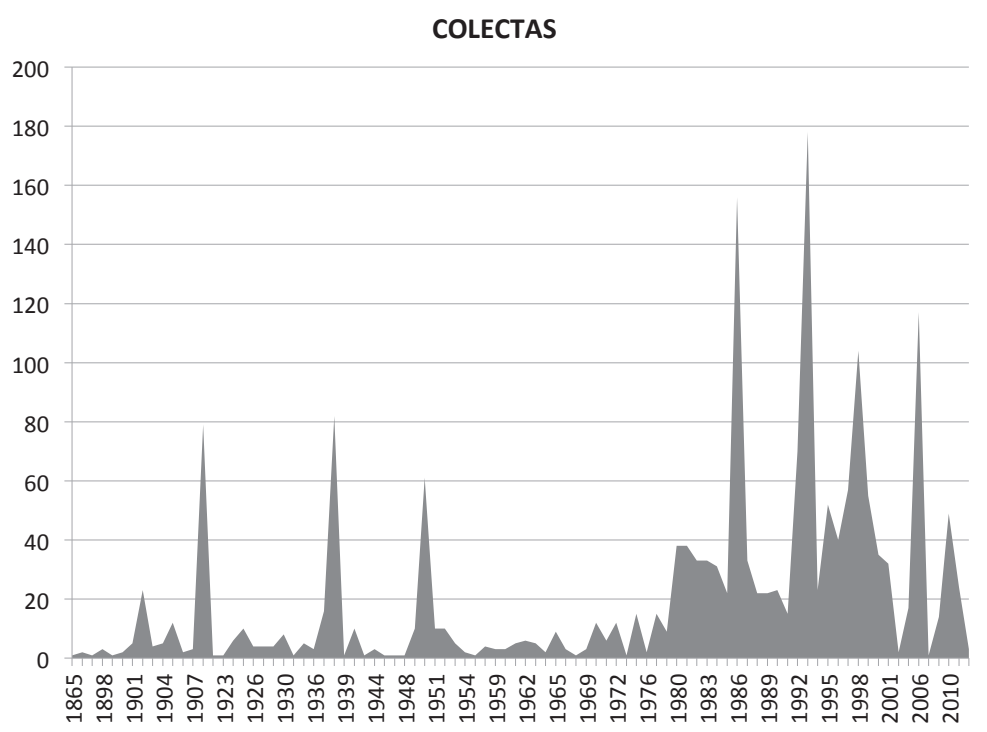

Fig. 1. Representación gráfica del esfuerzo de colecta a través de los años desde 1865 a la actualidad.

\section{COLECTAS}

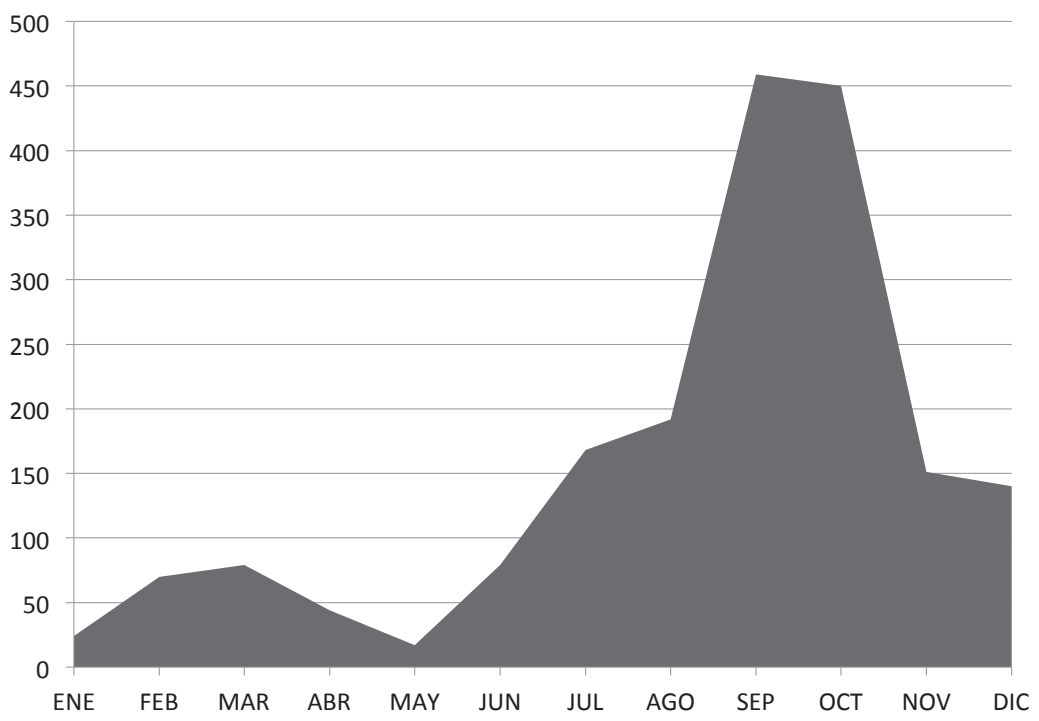

Fig. 2. Número de especímenes colectados en cada mes en el estado de Morelos. 
Cuadro 2. Municipios del estado de Morelos, su extensión geográfica, diversidad y número de especímenes de gramíneas colectados en cada uno. En negritas los municipios con el mayor número de colectas.

\begin{tabular}{|c|c|c|c|c|}
\hline Municipio & Géneros & Especies & Colectas & $\mathrm{km}^{2}$ \\
\hline Amacuzac (AMA) & 17 & 25 & 33 & 125 \\
\hline Atlatlahuacan (ATL) & 5 & 8 & 10 & 71 \\
\hline Axochiapan (AXO) & 24 & 39 & 62 & 143.1 \\
\hline Ayala (AYA) & 21 & 27 & 33 & 377.82 \\
\hline Coatlán del Río (COA) & 5 & 6 & 6 & 83.27 \\
\hline Cuautla (CUA) & 33 & 52 & 95 & 96.99 \\
\hline Cuernavaca (CUE) & 61 & 138 & 323 & 200.41 \\
\hline Emiliano Zapata (EMI) & 6 & 7 & 7 & 68.37 \\
\hline Huitzilac (HUI) & 41 & 83 & 195 & 191.18 \\
\hline Jantetelco (JAN) & 27 & 38 & 55 & 102.12 \\
\hline Jiutepec (JIU) & 21 & 29 & 40 & 55.49 \\
\hline Jojutla (JOJ) & 23 & 36 & 66 & 153.41 \\
\hline Jonacatepec (JON) & 10 & 10 & 13 & 90.27 \\
\hline Mazatepec (MAZ) & 9 & 11 & 13 & 57.81 \\
\hline Miacatlán (MIA) & 26 & 41 & 80 & 214.49 \\
\hline Ocuituco (OCU) & 20 & 23 & 29 & 86.91 \\
\hline Puente de Ixtla (PUE) & 23 & 37 & 49 & 297.43 \\
\hline Temixco (TEX) & 24 & 28 & 32 & 102.89 \\
\hline Temoac (TEM) & 7 & 9 & 12 & 37.04 \\
\hline Tepalcingo (TEP) & 20 & 38 & 78 & 367.67 \\
\hline Tepoztlán (TPO) & 42 & 85 & 162 & 252.87 \\
\hline Tetecala (TET) & 12 & 14 & 15 & 67.93 \\
\hline Tetela del Volcán (TEL) & 6 & 6 & 8 & 98.46 \\
\hline Tlalnepantla (TLN) & 28 & 42 & 64 & 109.62 \\
\hline Tlaltizapán (TLT) & 20 & 25 & 31 & 238.06 \\
\hline Tlaquiltenango (TLQ) & 29 & 41 & 78 & 543.59 \\
\hline Tlayacapan (TLY) & 33 & 59 & 105 & 57.33 \\
\hline Totolapan (TOT) & 21 & 26 & 42 & 60.08 \\
\hline Xochitepec (XOC) & 24 & 41 & 55 & 92.93 \\
\hline Yautepec (YAU) & 45 & 84 & 153 & 192.11 \\
\hline Yecapixtla (YEC) & 13 & 15 & 21 & 176.41 \\
\hline Zacatepec (ZAC) & 14 & 17 & 26 & 26.28 \\
\hline Zacualpan de Amilpas (ZCU) & 12 & 14 & 17 & 53.77 \\
\hline
\end{tabular}


Los municipios de Atlatlahucan, Coatlán del Río, Emiliano Zapata, Jonacatepec, Mazatepec, Temoac, Tetecala y Tetela del Volcán, que ocupan $11.73 \%$ de la extensión geográfica, son los menos estudiados ya que en total cuentan con sólo 84 especímenes, apenas $4.11 \%$ del número total. El resto de municipios tienen entre 20 y 95 especímenes cada uno. El municipio de Cuernavaca es el mejor estudiado con 323 especímenes distribuido en 61 géneros y 138 especies. Hay municipios como Tlayacapan cuya extensión geográfica de $57.33 \mathrm{~km}^{2}$ tiene un número de colectas de 105, mientras que otros como Tlaquiltenango con 543.59 $\mathrm{km}^{2}$, cuya extensión geográfica es la mayor del estado, sólo cuenta con 78 colectas. La Figura 3 muestra la representación gráfica de los datos presentados en el Cuadro 2.

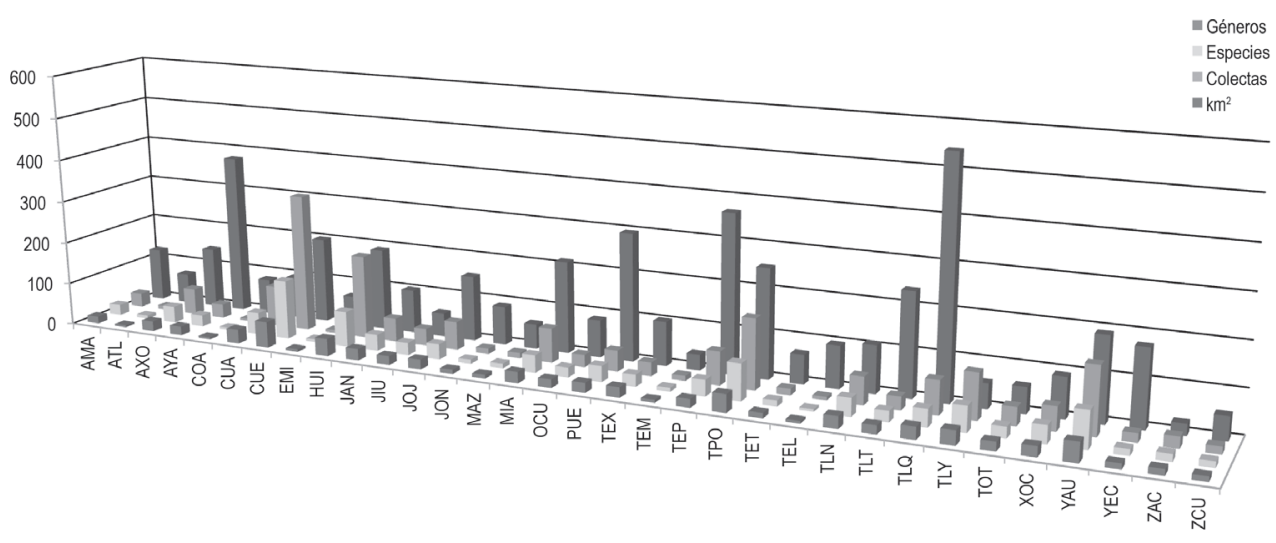

Fig. 3. Representación gráfica mostrando de adelante hacia atrás el número de géneros, especies, especímenes colectados y la extensión geográfica de cada uno de los municipios del estado de Morelos. Las abreviaciones de los municipios aparecen en el Cuadro 1.

La riqueza de géneros y especies de gramíneas se concentra en las subfamilias Pooideae (29/54), Chloridoideae (19/83) y Panicoideae (47/155), siendo ésta última la más diversa. Las tribus más ricas en géneros y especies son las Paniceae (19/76), Chlorideae (16/69) y Andropogoneae (22/46) (Cuadro 3).

El Cuadro 4 muestra a los géneros más diversos en el estado de Morelos, todos pertenecen a las subfamilias Panicoideae y Chloridoideae, siendo Muhlenbergia Schreb. y Paspalum L. los más diversos con 28 y 26 especies, respectivamente. 
Cuadro 3. Riqueza de la familia Poaceae en el estado de Morelos.

\begin{tabular}{llcc}
\hline Subfamilia & Tribu & Géneros & Especies \\
\hline Bambusoideae 3/3 & Bambuseae & 3 & 3 \\
Ehrhartoideae 2/2 & Oryzeae & 2 & 2 \\
Pooideae 29/54 & & & \\
& Brachypodieae & 1 & 2 \\
& Bromeae & 1 & 5 \\
& Hordeeae & 3 & 4 \\
& Meliceae & 2 & 3 \\
& Poeae & 18 & 34 \\
& Stipeae & 4 & 6 \\
Panicoideae 47/155 & & & \\
& Arundinelleae & 1 & 2 \\
& Paniceae & 19 & 76 \\
& Paspaleae & 4 & 29 \\
& Andropogoneae & 22 & 46 \\
Chloridoideae 19/83 & Zeugiteae & 1 & 2 \\
& Chlorideae & 16 & 69 \\
& Eragrostideae & 2 & 12 \\
& Zoysieae & 1 & 2 \\
Aristidoideae 1/6 & Aristideae & 1 & 6 \\
Arundinoideae 2/2 & Arundineae & 2 & 2 \\
Danthonioideae 1/1 & Danthonieae & 1 & 1 \\
& & 104 & 306 \\
\hline
\end{tabular}

El Cuadro 5 muestra el número de géneros y especies introducidas, nativas y cultivadas. Del total de géneros presentes, $44 \%$ tienen especies introducidas y $67 \%$ nativas. Existen géneros que pueden tener especies nativas e introducidas. A nivel específico, $24 \%$ son introducidas (73 especies), mientras que las restantes 233 son nativas. Casi la mitad de la flora genérica (47 géneros) tiene especies que son cultivadas, aunque no necesariamente están presentes en el estado de Morelos.

Entre las especies de malezas nocivas listadas en la NOM-043-FITO-1999 (2000) en el estado de Morelos, se encuentran siete especies (Cuadro 6). Existen 
Cuadro 4. Géneros con mayor riqueza de especies en el estado de Morelos.

\begin{tabular}{llc}
\hline Tribu & Género & Especies \\
\hline Chlorideae & Muhlenbergia Schreb. & 28 \\
Paspaleae & Paspalum L. & 26 \\
Chlorideae & Bouteloua Lag. & 18 \\
Paniceae & Digitaria Haller & 15 \\
Eragrostideae & Eragrostis Wolf & 11 \\
Paniceae & Cenchrus L. & 11 \\
Andropogoneae & Bothriochloa Kuntze & 9 \\
Paniceae & Urochloa P. Beauv. & 9 \\
Paniceae & Setaria P. Beauv. & 7 \\
Paniceae & Panicum L. & 6 \\
\hline
\end{tabular}

Cuadro 5. Riqueza de taxa introducidos, nativos y cultivados en el estado de Morelos.

\begin{tabular}{lcc}
\hline & Géneros & Especies \\
\hline Introducidas & 46 & 73 \\
Nativas & 70 & 233 \\
Cultivadas & 47 & 63 \\
Introducidas cultivadas & 33 & 43 \\
Nativas cultivadas & 14 & 20 \\
\hline
\end{tabular}

dos especies recién introducidas al estado y a México, una de ellas es Themeda quadrivalvis (L.) Kuntze que se considera muy agresiva (Sánchez-Ken et al., 2013). La otra es Hyparrhenia variabilis Stapf de África, recientemente descubierta para los estados de Jalisco y Michoacán, y para México (Vibrans et al., 2014). El listado florístico de la familia Poaceae en el estado de Morelos corrobora las especies de malezas nocivas citadas en el Catálogo de malezas nativas e introducidas de México (Sánchez-Ken et al., 2012).

Finalmente, la posición del estado de Morelos con respecto a su diversidad agrostológica a nivel nacional se modifica. Previamente, Bonilla-Barbosa y Villaseñor (2003) mencionaron que la familia Poaceae con 216 especies estaba ubicada en el tercer lugar de diversidad florística con respecto a los demás estados de la república. Sin embargo, éste número parece que sólo se refiere a taxa nativos ya 
Cuadro 6. Especies de gramíneas consideradas malezas nocivas en la NOM-043-FITO-1999 (2000) presentes en el estado de Morelos.

Malezas nocivas

Digitaria abyssinica (Hochst. ex. A. Rich.) Stapf (= D. scalarum (Schweinf.) Chiov.)

Digitaria velutina (Forssk.) P. Beauv.

Cenchrus polystachios (L.) Morrone (= Pennisetum polystachyum L.)

Setaria pumila (Poir.) Roem. \& Schult.

Urochloa panicoides P. Beauv.

Rottboellia cochinchinensis (Lour.) Clayton

Themeda quadrivalvis (L.) Kuntze

que en la lista presentan 93 géneros con 272 especies, con 22 categorías infraespecíficas. De ésta lista, cuatro especies están duplicadas con nombres inválidos y 61 nombres representan sinónimos o especies que han cambiado de nombre. Se mencionaron 46 nombres en la lista de los cuales no se encontró ningún espécimen de herbario, es probable que estos nombres sean producto de identificaciones erróneas. Por otro lado, el estado se consideró en la posición $20^{\text {a }}$ con 226 especies (Dávila et al., 2006), mientras que con los resultados obtenidos aquí se añaden 80 especies para un total de 306. Con este número de especies el estado de Morelos se posiciona en el lugar $13^{\circ}$ con respecto al resto de las entidades federativas. Es importante notar que esta posición probablemente puede variar de forma ascendente cuando se exploren de manera sistemática los municipios cuyo número de colectas es muy bajo.

\section{CONCLUSIONES}

Las conclusiones más importantes de este trabajo son las siguientes:

- La familia Poaceae está representada en el estado de Morelos por 104 géneros, 306 especies con 10 categorías infraespecíficas.

- La posición del estado de Morelos en cuanto a su riqueza agrostológica asciende a la posición $13^{\mathrm{a}}$ a nivel nacional.

- $\quad$ Finalmente, se concluye que existe la necesidad de realizar colectas sistematizadas de especímenes en los municipios pobremente colectados. 


\section{AGRADECIMIENTOS}

Agradecemos a los curadores de los herbarios HUMO, MEXU, UAMIZ y UAMX, por haber permitido revisar las colecciones botánicas.

\section{LITERATURA CITADA}

Bárcenas, A. 1977. La vegetación herbácea de Coatlán del Río, Morelos. Tesis de Licenciatura. Departamento de Biología, Facultad de Ciencias. Universidad Nacional Autónoma de México. México, D.F., México. 145 pp.

Bonilla-Barbosa, J. R. y A. J. Viana. 1997. Listado Florístico de México. XIV Flora del Parque Nacional Lagunas de Zempoala, México. Instituto de Biología, Universidad Nacional Autónoma de México. México, D.F., México. 31 pp.

Bonilla-Barbosa, J. R. y J. L. Villaseñor. 2003. Catálogo de la flora del Estado de Morelos. Universidad Autónoma del Estado de Morelos. Morelos, México. 129 pp.

Brummitt, R. K. and C. E. Powell. 1992. Authors of Plant Names. Royal Botanic Gardens, Kew. Kew, U.K. 736 pp.

Bustos M., O. Y. 2008. La subfamilia Chloridoideae (Poaceae) en el estado de Morelos, México. Tesis de Licenciatura. Escuela de Ciencias Biológicas. Universidad Autónoma del Estado de Morelos. Morelos, México. 145 pp.

Cerros-Tlatilpa, R. y A. Espejo-Serna. 1998. Contribución al estudio florístico de los cerros El Sombrerito y Las Mariposas (Zoapapalotl) en el Municipio de Tlayacapan, Morelos, México. Polibotánica 8: 29-46.

Contreras-MacBeath, T., J. R. Bonilla-Barbosa, J. C. Boyás-Delgado, G. Bustos-Zagal, J. M. Caspeta-Mandujano, R. Castro-Franco, M. A. Lozano-García, J. I. Martínez-Thomas, H. Mejía-Mojica, A. L. Ortiz-Villaseñor, D. Portugal-Portugal, R. Trejo-Albarrán, A. Trejo-Loyo y F. Urbina-Torres. 2004. Biodiversidad. In: Contreras-MacBeath, T., J. C. Boyas y F. Jaramillo (eds.). La diversidad biológica en Morelos: Estudio del estado. Comisión Nacional para el Conocimiento y Uso de la Biodiversidad y Universidad Autónoma del Estado de Morelos. Morelos, México. 156 pp.

Dávila, P., M. T. Mejía-Saulés, M. Gómez-Sánchez, J. Valdés-Reyna, J. J. Ortíz, C. Morín, J. Castrejón y A. Ocampo. 2006. Catálogo de las gramíneas de México. Universidad Nacional Autónoma de México-Comisión Nacional para el Conocimiento y Uso de la Biodiversidad. México, D.F., México. 671 pp.

Díaz-Pulido, C. I. 1998. Las gramíneas (Poaceae) forrajeras del municipio de Tepoztlán, estado de Morelos. Universidad Autónoma Metropolitana. México, D.F., México. 46 pp.

Díaz-Pulido, C. I., J. A. Chávez-Pérez, H. Weber-Díaz, E. L. Barriga-Martínez. 2007a. Las gramíneas (Poaceae) del municipio de Yautepec, Estado de Morelos. Universidad Autónoma Metropolitana. México, D.F., México. 72 pp.

Díaz-Pulido, C. I., J. A. Chávez-Pérez y H. Weber-Díaz. 2007b. Las Gramíneas (Poaceae) del municipio de Amacuzac, estado de Morelos. Universidad Autónoma Metropolitana. México, D.F., México. 52 pp. 
Díaz-Pulido, C. I., J. A. Chávez-Pérez, H. Weber-Díaz, B. A. Reyes-López. 2007c. Las Gramíneas (Poaceae) del municipio de Zacatepec, estado de Morelos. Universidad Autónoma Metropolitana. México, D.F., México. 56 pp.

Díaz-Pulido, C. I., M. R. Granados-Agustin, H. Weber-Díaz. 2007d. Las Gramíneas (Poaceae) del municipio de Ocuituco, estado de Morelos. Universidad Autónoma Metropolitana. México, D.F., México. 150 pp.

Díaz-Pulido, C. I., M. R. Granados-Agustin, J. A. Chávez-Pérez y H. Weber-Díaz. 2007e. Las Gramíneas (Poaceae) del municipio de Axochiapan, estado de Morelos. Universidad Autónoma Metropolitana. México, D.F., México. 93 pp.

Dorado, O., B. Maldonado, D. M. Arias, V. Sorani, R. Ramírez, E. Leyva y D. Valenzuela. 2005. Programa de Conservación y Manejo Reserva de la Biosfera de Huautla. Comisión Nacional de áreas Naturales Protegidas-Secretaría de medio Ambiente y Recursos Naturales. México, D.F., México. 143 pp.

Flores-Castorena, A. y D. Martínez-Alvarado. 2011. Capítulo V. Sinopsis Florística. In: Bonilla-Barbosa, J., M. V. Mora, J. Luna-Figueroa, H. Colín y S. Santillán-Alarcón (eds.). Biodiversidad, conservación y manejo en el Corredor Biológico Chichinautzin condiciones actuales y prespectivas. Universidad Autónoma del Estado de Morelos. Morelos, México. pp. 69-97.

Galindo-Becerril, G. y R. Fernández-Nava. 2002. Inventario florístico del municipio de Amacuzac, Morelos, México. Polibotánica 13: 107-135.

González-Barrales, M. N. 2006. Té Limón (Cymbopogon citratus Stapf). Jardín Botánico de la Universidad Autónoma del Estado de Morelos. Tlahui-Medic 21: 1.

INEGI. 2013. Perspectiva estadística Morelos. Instituto Nacional de Estadística y Geografía. México, D.F., México. 93 pp.

Itie, G. 1935. Estudio de las gramíneas de la región del Tepozteco, Morelos. Boletín de la Sociedad Botánica de México 5: 20-26.

March-Mifsut, J. y M. Martínez-Jiménez. 2007. Especies invasoras de alto impacto a la biodiversidad. Prioridades en México. Instituto Mexicano de Tecnología del Agua. México, D.F., México. 72 pp.

NOM-043-FITO-1999. 2000. Especificaciones para prevenir la introducción de malezas cuarentenarias a México. Diario Oficial, Primera Sección. México, D.F. México. 4 pp.

Pulido-Esparza, V. A., A. Espejo-Serna y A. R. López-Ferrari. 2009. Las monocotiledóneas nativas del corredor biológico Chichinautzin. Acta Botanica Mexicana 86: 9-38.

Rzedowski, J. 1978. Vegetación de México. Limusa. México, D.F., México. 432 pp.

Sánchez-Ken, J. G., R. Cerros-Tlatilpa y H. Vibrans. 2013. Themeda quadrivalvis (Sacchareae, Panicoideae, Poaceae), una maleza reglamentada presente y establecida en el estado de Morelos, México. Botanical Sciences 91(4): 531-536.

Sánchez-Ken, J. G., G. A. Zita-Padilla y M. Mendoza-Cruz. 2012. Catálogo de malezas gramíneas nativas e introducidas de México. Consejo Nacional Consultivo Fitosanitario. México, D.F. México. 433 pp.

Soreng, R. J., G. Davidse, P. M. Peterson, F. O. Zuloaga, E. J. Judziewicz, T. S. Filgueiras and O. Morrone. 2003 (and onwards). Catalogue of New World Grasses. Available from: www.tropicos.org/Project/CNWG (actualizado en 2012). 
Soreng, J. J., P. M. Peterson, K. Romaschenko, G. Davidse, F. O. Zuloaga, E. J. Judziewicz, T. S. Filgueiras, J. I. Davis and O. Morrone. 2015. A worldwide phylogenetic classification of the Poaceae (Gramineae). Journal of Systematics and Evolution 53(2): 117-137.

Soria, R. G. 1985. Flora de Morelos. Descripción de especies vegetales de la Selva baja caducifolia del Cañón de Lobos. Mpio. de Yautepec. Programa florístico-ecológico Coordinación de Investigación. Universidad Autónoma del Estado de Morelos. Morelos, México. 163 pp.

Sotelo, G. A. 1984. La subfamilia Panicoideae (Gramineae) en el estado de Morelos. Tesis de Licenciatura. Escuela de Ciencias Biológicas. Universidad Autónoma del Estado de Morelos. Morelos, México. 221 pp.

Vázquez, S. J. 1974. Contribución al estudio de las plantas del Estado de Morelos (México). Catálogo de las plantas contenidas en el "Herbario L'Amagatall". Ciencia 29: 1-138.

Vibrans, H., E. García-Moya, D. Clayton y J. G. Sánchez-Ken. 2014. Hyparrhenia variabilis and Hyparrhenia cymbaria (Poaceae): new for the Americas, successful in Mexico. Invasive Plant Science Management 7(2): 222-228. 


\section{APÉNDICE 1}

Literatura consultada para la elaboración del listado de la familia Poaceae.

Beetle, A. A., R. Guzmán-Mejía, V. Jaramillo-Luque, M. P. Guerrero-Sánchez, E. ManriqueForceck, A. Chimal-Hernández, C. Shariff-Bujdud y I. Núñez-Tancredi. 1983. Las Gramíneas de México. Vol. I. Secretaría de Agricultura y Recursos HidráulicosCOTECOCA. México, D.F., México. 260 pp.

Beetle, A. A., E. Manrique-Forceck, V. Jaramillo-Luque, M. P. Guerrero-Sánchez, J. A. Miranda-Sánchez, I. Núñez-Tancredi y A. Chimal-Hernández. 1987. Las Gramíneas de México. Tomo II. Secretaría de Agricultura, Ganadería y Desarrollo Rural. México, D.F., México. 335 pp.

Beetle, A. A., E. Manrique-Forceck, J. A. Miranda-Sánchez, V. Jaramillo-Luque, A. Chimal-Hernández y A. M. Rodríguez-Rodríguez. 1991. Las Gramíneas de México. Vol. III. Secretaría de Agricultura y Recursos Hidráulicos-COTECOCA. México, D.F., México. 332 pp.

Beetle, A. A., J. A. Miranda-Sánchez, V. Jaramillo-Luque, A. M. Rodríguez-Rodríguez, L. Aragón-Melchor, M. A. Vergara-Batalla, A. Chimal-Hernández y O. DomínguezSepúlveda. 1995. Las Gramíneas de México. Vol. IV. Secretaría de Agricultura y Recursos Hidráulicos-COTECOCA. México, D.F., México. 372 pp.

Beetle, A. A., G. Villegas-Durán, A. Bolaños-Medina A., J. A. Miranda-Sánchez, L. AragónMelchor, M. A. Vergara-Batalla, A. Chimal-Hernández, M. M. Castillo-Badillo, O. M. Galván-García, J. L. Villalpando-Prieto, M. Lizama-Manrique, J. Valdés-Reyna, E. Manrique-de-Skendzic y A. M. Rodríguez-Rodríguez. 1999. Las Gramíneas de México. Vol. V. Secretaría de Agricultura y Recursos Hidráulicos-COTECOCA. México, D.F., México. 482 pp.

Clayton, W. D., M. S. Vorontsova, K. T. Harman and H. Williamson. 2006 and onwards. GrassBase - The Online World Grass Flora. www.kew.org/data/grasses-db.html. [accessed 10 Nov. 2011].

McVaugh, R. 1983. Gramineae. A descriptive account of the vascular plants of western Mexico. Flora Novo-Galiciana 14: 1-436.

Peterson, P. M., K. Romaschenko and Y. Herrera A. 2015. A molecular phylogeny and classification of the Eleusininae with a new genus, Micrachne (Poaceae: Chloridoideae: Cynodonteae). Taxon 64(3): 445-467.

Peterson, P. M., R. J. Soreng, G. Davidse, T. S. Filgueiras, F. O. Zuloaga and E. J. Judziewicz. 2001. Catalogue of New World Grasses (Poaceae): II. Subfamily Chloridoideae. Contributions from The United States National Herbarium 41: 1-225.

Saarela, J. M., P. M. Peterson and J. Valdés-Reyna. 2014. A taxonomic revisión of Bromus (Poaceae: Pooideae: Bromeae) in Mexico and Central America. Phytotaxa 185(1): $1-147$.

Sánchez-Ken, J. G. 2010. Two new species of Paspalum (Paniceae: Panicoideae: Poaceae), a preliminary checklist of the genus in Mexico, and the identity of $P$. crinitum. Revista Mexicana de Biodiversidad 81: 629-647. 
Apéndice 1. Continuación.

Sánchez-Ken, J. G. 2011a. Two new species of Urochloa (Paniceae; Panicoideae; Poaceae) from Western Mexico and the updated checklist with a key to species of the genus in Mexico. Systematic Botany 36(3): 621-630.

Sánchez-Ken, J. G. 2011b. Poaceae: Subfamilia Panicoideae. Flora del Valle de TehuacánCuicatlán 81: 1-223.

Sánchez-Ken, J. G. 2012. A synopsis of Digitaria (Paniceae, Panicoideae, Poaceae) in Mexico, including the new species Digitaria michoacanensis. Acta Botanica Mexicana 101: 127-149.

Soreng, R. J., G. Davidse, P. M. Peterson, F. O. Zuloaga, E. J. Judziewicz, T. S. Filgueiras and O. Morrone. 2003 (and onwards). Catalogue of New World Grasses. Available from: www.tropicos.org/Project/CNWG (actualizado en 2012).

Soreng, R. J. and P. M. Peterson. 2012. Revision of Poa L. (Poaceae, Pooideae, Poeae, Poinae) in Mexico: new records, re-evaluation of $P$. ruprechtii, and two new species, P. palmeri and P. wendtii. PhytoKeys 15: 1-104.

Zuloaga, F. O., O. Morrone, G. Davidse, T. S. Filgueiras, P. M. Peterson, R. J. Soreng and E. Judziewicz. 2003. Catalogue of New World Grasses (Poaceae): III. Subfamilies Panicoideae, Aristidoideae, Arundinoideae, and Danthonioideae. Contribution from The United States National Herbarium 46: 1-662. 


\section{APÉNDICE 2}

Listado florístico de la familia Poaceae en el estado de Morelos. El orden del listado es por subfamilia, numérico y alfabético, cada número está compuesto del nombre de la especie, autor(es), seguido por el(los) colector(es), el número de colecta y el(los) herbario(s) donde se encuentra depositado el espécimen. Cuando hay varios números de colecta por el mismo colector pero depositados en diferentes herbarios, sólo se separan con comas con sus respectivos herbarios. Al final de cada nombre se presentan algunos atributos de las especies con respecto a su estatus, usos y se especifica si es cultivada. Nat.=Nativa, Int. $=$ Introducida. Atributos: a. alimento, c. cereal, ce. control de erosión, f. forraje, m. maleza, me. medicinal, mn. maleza nociva, mp. maleza potencial, o. ornamental, op. ornamental potencial, r. ruderal, s. silvestre, u. varios usos. Cult. $=$ Cultivada.

\section{Aristidoideae Caro}

1. Aristida adscensionis L. - C. Aguilar R. \& C. Díaz P. s.n. (UAMX); S. Casado A. \& C. Díaz P. s.n. (UAMX); R. Cerros T. 1610 (UAMIZ); M. Chávez M. \& C. Díaz P. s.n. (UAMX); P. Corrales A. \& C. Díaz P. s.n. (UAMX); C. Díaz P. s.n. (UAMX); C. Díaz P. et al. 3, 120 (UAMX); C.A. Fuentes T. \& C. Díaz P. s.n. (UAMX); A. García C. \& C. Díaz P. s.n. (UAMX); M. García G. \& C. Díaz P. s.n. (UAMX); R. García \& C. Díaz P. s.n. (UAMX); S. Gaytán O. et al. 393, 405, s.n. (UAMX); J. Gutiérrez \& C. Díaz P. s.n. (UAMX); A.S. Hitchcock 6865 (US); E. Luna C. \& C. Díaz P. s.n. (UAMX); E. Lyonnet 2641 (MO); J.L. Marín V. \& C. Díaz P. s.n. (UAMX); A.S. Martínez G. \& C. Díaz P. s.n. (UAMX); G. Martínez S. \& C. Díaz P. s.n. (UAMX); M. Moyers R. \& C. Díaz P. s.n. (UAMX); G. Naranjo \& C. Díaz P. s.n. (UAMX); A. Quiñones R. \& C. Díaz P. s.n. (UAMX); A.E. Reséndiz C. \& C. Díaz P. s.n. (UAMX); C. Rojas L. \& C. Díaz P. s.n. (UAMX); H. Rufino \& C. Díaz P. s.n. (UAMX); sin colector s.n. (UAMX); D.N. Trejo M. \& C. Díaz P. s.n. (UAMX); H. Vite P. \& C. Díaz P. s.n. (UAMX). Nat., r.

2. Aristida appressa Vasey - E.W.D. Holway 3020 (US); E. Lyonnet 2428 (MO, US). Nat., r.

3. Aristida jorullensis Kunth - R. Cerros T. 1543 (HUMO). Nat., r., s.

4. Aristida laxa Cav. - J. Sánchez B. IA (MEXU). Nat., r.

5. Aristida schiedeana Trin. \& Rupr. - W. Celis C. \& C. Díaz P. s.n. (UAMX); R. Cerros T. 103 (UAMIZ); M. Espinoza M. \& C. Díaz P. s.n. (UAMX); R.A. Hernández C. et al. 34, 233, 282, 580 (HUMO, UAMIZ); E. Lyonnet 633, 1355, 1436, 2423 (US); F. Millán A. \& C. Díaz P. s.n. (UAMX); C.R. Orcutt 3863 (MO, US); F. Robles P. \& C. Díaz P. s.n. (UAMX); E. Rodríguez P. \& C. Díaz P. s.n. (UAMX); O. Rojas M. et al. s.n. (UAMX); A. Soberanes \& C. Díaz P. s.n. (UAMX); J. Vázquez E. \& C. Díaz P. s.n. (UAMX). Nat., r.

6. Aristida ternipes Cav. - R.E. Aguilar H. et al. s.n. (UAMX); J.J. Aguirre P. \& C. Díaz P. s.n. (UAMX); E. Bourgeau 1295 (US); G. Carranza A. \& C. Díaz P. s.n. (UAMX); M.I. 
Apéndice 2. Continuación.

Delgado R. \& C. Díaz P. s.n. (UAMX); C. Díaz P. s.n. (UAMX); C. Díaz P. \& O. Rojas M. s.n. (UAMX); C. Díaz P. et al. 113 (UAMX); E. Estrada L. 1394 (UAMIZ); M.A. Flores V. \& C. Díaz P. s.n. (UAMX); M.L. Gallardo R. \& C. Díaz P. s.n. (UAMX); M. García G. \& C. Díaz P. s.n. (UAMX); S. García Z. \& C. Díaz P. s.n. (UAMX); C. Gatica S. \& C. Díaz P. s.n. (UAMX); S. Gaytán O. et al. 81, 391, 420, s.n. (UAMX); M. González \& C. Díaz P. s.n. (UAMX); M.M. Hernández A. \& C. Díaz P. s.n. (UAMX); R.I. Hernández A. \& C. Díaz P. s.n. (UAMX); A.S. Hitchcock 6831 (US); R. Iglesias et al. s.n. (UAMX); F. Jiménez O. \& C. Díaz P. s.n. (UAMX); J.G. Jiménez V. et al. s.n. (UAMX); R.M. Lagunas M. \& C. Díaz P. s.n. (UAMX); M.R.N.A. \& C. Díaz P. s.n. (UAMX); M.S. Macías L. \& C. Díaz P. s.n. (UAMX); J.L. Marín V. \& C. Díaz P. s.n. (UAMX); R. Mendoza M. \& C. Díaz P. s.n. (UAMX); G. Naranjo C. Díaz P. s.n. (UAMX); A. Olguín G. \& C. Díaz P. s.n. (UAMX); V. Ortega C. \& C. Díaz P. s.n. (UAMX); C.G. Pringle 6496 (MO); C. Reséndiz \& C. Díaz P. s.n. (UAMX); J.N. Rose et al. 10227 (US); H. Rufino \& C. Díaz P. s.n. (UAMX); sin colector s.n. (UAMX); R. Téllez G. \& C. Díaz P. s.n. (UAMX); D.N. Trejo M. \& C. Díaz P. s.n. (UAMX); V.Q.P.L. \& C. Díaz P. s.n. (UAMX); M.E. Vela G. \& C. Díaz P. s.n. (UAMX); E. Vicente de la C. \& C. Díaz P. s.n. (UAMX). Nat., r.

Arundinoideae Kunth ex Beilschm.

7. Arundo donax L. - March-Mifsut y Martínez-Jiménez (2007). Int., m., r., o., u. Cult.

8. Phragmites australis (Cav.) Steud. - J. Vázquez S. 1525, 1743 (MEXU). Int., m., r., o., u. Cult.

Bambusoideae Luerss.

9. Bambusa vulgaris Schrad. ex J.C. Wendl. - A. Bonfil 209 (UAMIZ); C.G. Pringle 7163 (MO, US); G.B. Ross s.n. (US); sin colector 46 (UAMX); J. Vázquez S. 722 (MEXU). Int., r., o., u. Cult.

10. Otatea acuminata (Munro) C.E. Calderón \& Soderstr. subsp. aztecorum Guzmán, Anaya \& Santana - R. Cerros T. et al. 2929 (HUMO, MEXU); J. Vázquez S. 2147, 2551 (MEXU). Nat., r., o., u.

11. Phyllostachys aurea Carrière ex Rivière \& C. Rivière - R.A. Gómez C. s.n. (UAMX). Int., o. Cult.

Chloridoideae Kunth ex Beilschm.

12. Bouteloua aristidoides (Kunth) Griseb. - A. Bonfil 162 (UAMIZ); de la Mora 524 (MEXU, UAMIZ). Nat., r.

13. Bouteloua barbata Lag. - C.E. Boyd 48 (US); J.T. Camp 46 (HUMO); G. Davidse 35580 (MO); A. González \& B. Rodríguez s.n. (HUMO); S.D. Koch 75313 (US); J. Morales O. 129 (HUMO). Nat., r.

14. Bouteloua curtipendula (Michx.) Torr. - J.G. Aldape et al. 83 (HUMO); S. Cacho 8 (HUMO); P. Dávila A. 4 (HUMO); G. Itié s.n. (HUMO); E. Manrique et al. s.n. (HUMO); de la Mora 523 (HUMO); J. Morales O. $176 a$ (HUMO); V. Peñaloza G. 853 (HUMO). Nat., f., r. Cult.

15. Bouteloua dactyloides (Nutt.) Columbus - E. Lyonnet 2621 (MO, US); J.R. Reeder \& C.G. Reeder 2210 (HUMO). Nat., ce., f., r. Cult. 
Apéndice 2. Continuación.

16. Bouteloua dimorpha Columbus - J.J. Aguirre P. \& C. Díaz P. s.n. (UAMX); M.C. Avella M. 5 (HUMO); N. Baños D. \& C. Díaz P. s.n. (UAMX); A. Bonfil 146 (UAMIZ); S. Cacho 40 (MEXU, UAMIZ), s.n. (HUMO); E. Camacho D. \& C. Díaz P. s.n. (UAMX); S. Casado A. \& C. Díaz P. s.n. (UAMX); E. Cervantes s.n. (UAMIZ); J.A. Chávez P. \& C. Díaz P. s.n. (UAMX); M. Chávez V. \& C. Díaz P. s.n. (UAMX); R. Cuevas E. \& C. Díaz P. s.n (UAMX); G. Davidse 35578, 35579 (MO); C. Díaz P. s.n. (UAMX); C. Díaz P. et al. s.n. (UAMX); O. Domínguez S. 106 (HUMO); P. Dorelus P. \& C. Díaz P. s.n. (UAMX); M. García G. \& C. Díaz P. s.n. (UAMX); S. Gaytán O. \& C. Díaz P. s.n. (UAMX); S. Gaytán O. et al. 299, s.n. (UAMX); F. González M. \& R. Dirzo 16 (HUMO); M. González \& C. Díaz P. s.n. (UAMX); F.W. Gould 10321 (US); J. Gutiérrez \& C. Díaz P. s.n. (UAMX); M.M. Hernández A. \& C. Díaz P. s.n. (UAMX); R.I. Hernández A. \& J.L. Mendoza G. s.n. (UAMX); A.S. Hitchcock 6817, 6842 (US); G. Itié s.n. (HUMO); A.W. Jackson 61 (HUMO); R.M. Lagunas M. \& C. Díaz P. s.n. (UAMX); J. Lobato s.n. (UAMIZ); O. López A. 65 a (HUMO); E. Lyonnet s.n. (MO); M.S. Macías L. \& C. Díaz P. s.n. (UAMX); J.L. Marín V. \& C. Díaz P. s.n(UAMX); A. Miranda S. \& P. Guerrero 364, 377 (HUMO); J.L. Monsivais M. 5 (UAMIZ); de la Mora 537 (HUMO); A.F. Morales J. \& C. Díaz P. s.n. (UAMX); J. Morales O. 92, 103, 109, 147 (HUMO); J. Morales \& O. López 51, 64, 65 (HUMO); M. Núñez M. 34, 40 (HUMO); C.G. Pringle 11237 (MO, US); O. Rodríguez T. s.n. (HUMO); D. Rojas F. \& C. Díaz P. s.n. (UAMX); H. Rufino \& C. Díaz P. s.n. (UAMX); J. Rzedowski 21452 (HUMO); sin colector s.n (UAMX); I.L. Soto M. \& C. Díaz P. s.n. (UAMX); M. Trujillo B. \& C. Díaz P. s.n. (UAMX); J. Vázquez S. 2712, 2713 (HUMO); G. Villegas 1625a (HUMO); M. Yepes s.n. (UAMIZ). Nat., r.

17. Bouteloua disticha (Kunth) Benth. - J.R. Reeder \& C.G. Reeder 4150 (US). Nat., r.

18. Bouteloua diversispicula Columbus - J.M. García \& A.C. Díaz P. s.n. (UAMX); S. Gaytán O. et al. 264, 386 (UAMX); M. Joseph C. \& C. Díaz P. s.n. (UAMX); O. López A. 54 (HUMO); J.L. Marín V. \& C. Díaz P. s.n. (UAMX); O. Martínez R. \& C. Díaz P. s.n. (UAMX); E. Medina G. \& C. Pérez D. s.n (UAMX); J. Morales O. 142 (UAMIZ); H. Rufino \& C. Díaz P. s.n. (UAMX); sin colector s.n. (UAMX); E. Vicente de la C. \& C. Díaz P. s.n. (UAMX). Nat., r.

19. Bouteloua erecta (Vasey \& Hack.) Columbus - C.J. Lyons Jr. 60 (US); P.P. Nye 63 (US); J.R. Walther 108 (US). Nat., r.

20. Bouteloua griffithsii Columbus - C.G. Pringle 8707 (MO, US). Nat., r.

21. Bouteloua hirsuta Lag. - A. Bonfil 403 (UAMIZ); J. Morales O. 121 (HUMO); J.N. Rose \& J.S. Rose 11055 (US); J. Vázquez S. 2629 (HUMO). Nat., f., r. Cult.

22. Bouteloua media (E. Fourn.) Gould \& Kapadia - F.W. Gould 10323 (US); A.S. Hitchcock 6862 (US); L. Pacheco \& P. Dávila A. 60 (MO); C.G. Pringle 11217 (MO, US). Nat., f., r.

23. Bouteloua multifida (Griffiths) Columbus - E. Lyonnet 2627 (US); J.R. Reeder 4400 (US); A.J. Sharp 441357 (US). Nat., r.

24. Bouteloua polymorpha (E. Fourn.) Columbus - M.C. Avella M. 3 (HUMO); R. Cerros T. \& M. Flores C. 199 (HUMO); F. González M. \& R. Dirzo 22 (HUMO); E. Lyonnet 627 (HUMO); E. Matuda 26314 (HUMO); J. Vázquez S. 3667 (HUMO). Nat., r. 
Apéndice 2. Continuación.

25. Bouteloua radicosa (E. Fourn.) Griffiths - M.C. Avella M. 11 (HUMO); W. Boege 1607 (HUMO); E. Estrada L. 1813 (UAMIZ); F. González M. \& R. Dirzo $5 a$ (HUMO); S.D. Koch 75316 (MO); E. Matuda 26320 (HUMO); J. Morales \& O. López 76 (HUMO); J. Vázquez S. 2632, 3531 (HUMO). Nat., r.

26. Bouteloua repens (Kunth) Scribn. \& Merr. - A. Bonfil 77 (UAMIZ); R. Cerros T. 131, 1600 (UAMIZ); F. González M. \& R. Dirzo 5, 30 (HUMO); F.W. Gould 10322 (US); A.S. Hitchcock 6820 (US); C.L. Lundell A. \& A. Lundell 12504 (US); E. Lyonnet 625, 2643 (MO, US); C.J. Lyons Jr. 113 (HUMO, MO); A. Miranda S. \& P. Guerrero 345 (HUMO); J. Morales O. 114, 126 (HUMO); M. Núñez M. 9, 31 (HUMO); P.P. Nye 30 (US); C.R. Orcutt 3885 (MO, US); J.N. Rose et al. 10189 (US). Nat., r.

27. Bouteloua simplex Lag. - G. Ayala A. 80 (HUMO). Nat., r.

28. Bouteloua triaena (Trin. ex Spreng.) Scribn. - J.G. Aldape et al. 86 (MEXU, MO, UAMIZ); J.L. Berlandier 1016 (US); J. Crutchfield \& M.C. Johnston 5975 (US); E. Estrada L. 1396 (UAMIZ); O. López A. 57 (MEXU, UAMIZ); E. Lyonnet \& J. Elcoro 1176 (US); A. Miranda S. \& P. Guerrero 363 (MEXU, UAMIZ); J. Morales O. 140, 176 (MEXU, UAMIZ); J. Morales O. \& López 54 (MEXU, UAMIZ); M. Núñez M. 99 (MEXU, UAMIZ); C.G. Pringle 8708 (MO, US); I. Rivera 19 (UAMIZ); G. Woronow 2392 (US). Nat., r.

29. Bouteloua williamsii Swallen - E. Blanco 24 (MEXU, UAMIZ). Nat., r.

30. Chloris gayana Kunth - A. Bastida T. \& C. Díaz P. s.n. (UAMX); W. Celis C.\& C. Díaz P. s.n. (UAMX); C. Díaz P. s.n. (UAMX); O. López A. 47 (HUMO); E. Lyonnet 2807 (MEXU, MO, UAMIZ, US); F. Morales J. \& C. Díaz P. s.n. (UAMX); E. Rubio de S. \& C. Díaz P. s.n. (UAMX); L.A. Vázquez H. \& C. Díaz P. s.n. (UAMX). Int., ce., f., mp. Cult.

31. Chloris pycnothrix Trin. - Y.E. López J. \& C. Díaz P. s.n. (UAMX); L. Morales s.n. (HUMO); V.H. Romero M. \& C. Díaz P. s.n. (UAMX); I.L. Soto M. \& C. Díaz P. s.n. (UAMX). Nat., r.

32. Chloris rufescens Lag. - V. Bueno F. \& C. Díaz P. s.n. (UAMX); R. Cerros T. 1603 (HUMO); C. Díaz P. s.n. (UAMX); C. Gatica S. \& C. Díaz P. s.n. (UAMX); M.C. Hernández L. \& C. Díaz P. s.n. (UAMX); A.S. Hitchcock 6855, 6875 (US); F. Martínez P. \& C. Díaz P. s.n. (UAMX); A. Miranda S. \& P. Guerrero 347, 374 (HUMO); H. Olvera A. \& C. Díaz P. s.n. (UAMX); D. Orozco P. \& C. Díaz P. s.n. (UAMX); C. Pérez D. \& C. Díaz P. s.n. (UAMX); J. Vázquez E. \& C. Díaz P. s.n. (UAMX). Nat., r.

33. Chloris submutica Kunth - M. Reyes B. 1171, 1175 (HUMO). Nat., r.

34. Chloris virgata Sw. - A. Aguilar V. \& C. Díaz P. s.n. (UAMX); M.C. Avella M. 59 (HUMO); A. Ayaga V. \& C. Díaz P. s.n. (UAMX); E. Bautirto J. \& C. Díaz P. s.n. (UAMX); M. Beltrán G. \& C. Díaz P. s.n. (UAMX); A. Bonfil 108 (HUMO); O. Bruno B. \& C. Díaz P. s.n. (UAMX); E. Camacho D. \& C. Díaz P. s.n. (UAMX); S. Casado A. \& C. Díaz P. s.n. (UAMX); E.M. Castillo R. \& C. Díaz P. s.n. (UAMX); R. Cerros T. 1602 (HUMO); J.A. Chávez P. \& C. Díaz P. s.n. (UAMX); M.A. Crispín I. \& V.M. Ortiz A. s.n. (UAMX); A. Cruz G. \& C. Díaz P. s.n. (UAMX); A. del Angel M. \& S. 
Apéndice 2. Continuación.

Hernández s.n. (UAMX); C. Díaz P. s.n. (UAMX); C. Díaz P. et al. s.n. (UAMX); D. Elías M. \& C. Díaz P. s.n. (UAMX); J.L. Espitia 2 (HUMO); M.A. Flores V. \& C. Díaz P. s.n. (UAMX); F. García de la C. \& C. Díaz P. s.n. (UAMX); M. García G. \& C. Díaz P. s.n. (UAMX); D. García P. \& C. Díaz P. s.n. (UAMX); L. Garduño A. \& C. Díaz P. s.n. (UAMX); S. Gaytán O. \& C. Díaz P. s.n. (UAMX); M. González \& C. Díaz P. s.n. (UAMX); I. González A. \& P. del Razo R. s.n. (UAMX); F. González M. \& R. Dirzo 28 (HUMO); S. Hernández \& C. Díaz P. s.n. (UAMX); P. Hernanio A. \& C. Díaz P. s.n. (UAMX); M.A. Herrera S. \& C. Díaz P. s.n. (UAMX); A.S. Hitchcock 6835 (US); A. Huchin G. \& C. Díaz P. s.n. (UAMX); A. Ibarra \& C. Díaz P. s.n. (UAMX); A.W. Jackson 70, 99 (HUMO); E. Jacobo A. \& C. Díaz P. s.n. (UAMX); S.E. Jean F. \& C. Díaz P. s.n. (UAMX); V. Jiménez et al. s.n. (UAMX); E. Lima \& C. Díaz P. s.n. (UAMX); O. López A. 36 (HUMO); O. López A. \& C. Díaz P. s.n. (UAMX); Y.E. López J. \& C. Díaz P. s.n. (UAMX); E. Lyonnet 2660 (MO, US), 2804 (US), 3066 (MEXU, MO, UAMIZ); C.J. Lyons Jr. 58 (HUMO), 112 (MO); M.S. Macías L. \& C. Díaz P. s.n. (UAMX); J. Maldonado 27, s.n. (HUMO); J.L. Marín V. \& C. Díaz P. s.n. (UAMX); J. Martineau J. \& R. Jaques G. s.n. (UAMX); J. Martínez C. \& C. Díaz P. s.n. (UAMX); E. Matuda 26315 (MO); V. Méndez R. \& C. Díaz P. s.n. (UAMX); N.M. Morales A. \& C. Díaz P. s.n. (UAMX); V.H. Moreno S. \& C. Díaz P. s.n. (UAMX); G. Naranjo \& C. Díaz P. s.n. (UAMX); I. Navarro C. \& C. Díaz P. s.n. (UAMX); M. Núñez M. 18 (HUMO); C.R. Orcutt s.n. (US); B. Osorio R. \& C. Díaz P. s.n. (UAMX); F. Prieta S. \& C. Díaz P. s.n. (UAMX); M. Pucheta D. s.n. (UAMX); M. Quezada R. 1342 (HUMO); G. Quintero L. \& C. Díaz P. s.n. (UAMX); G. Ramírez A. \& C. Díaz P. s.n. (UAMX); C. Ríos C. \& C. Díaz P. s.n. (UAMX); E. Rubio de S. \& C. Díaz P. s.n. (UAMX); H. Rufino \& C. Díaz P. s.n. (UAMX); G. Sánchez M. \& C. Díaz P. s.n. (UAMX); M.A. Sánchez M. \& C. Díaz P. s.n. (UAMX); E. Solís \& C. Díaz P. s.n. (UAMX); I.L. Soto M. \& C. Díaz P. s.n. (UAMX); R. Téllez G. \& C. Díaz P. s.n. (UAMX); J. Vázquez S. 1802 (HUMO); M.E. Vela G. \& C. Díaz P. s.n. (UAMX); J.L. Villa R. \& C. Díaz P. s.n. (UAMX). Nat., m., r. Cult.

35. Cynodon dactylon (L.) Pers. - M.C. Avella M. 22 (HUMO); C.E. Boyd 52 (HUMO); S. Cacho 45 (HUMO); J.T. Camp 45 (HUMO); A. Díaz O. 17 (HUMO); W. Fosberg 98 (HUMO); O. Gallardo C. s.n. (HUMO); T. Hunt 123 (HUMO); A.W. Jackson 66 (HUMO); O. López A. 4, 90, s.n. (HUMO); C.J. Lyons Jr. 114 (HUMO); J. Maldonado s.n. (HUMO); V. Mejía U. s.n. (HUMO); J. Morales O. 83, 110 (HUMO); M. Núñez M. 90 (HUMO); A. Pulido E. et al. 39 (HUMO); M. Quezada R. 1873 (HUMO); M. Reyes B. s.n. (HUMO); A. de los Santos 25, s.n. (HUMO); A. Sotelo G. 2706, s.n. (HUMO); C.H. Thomas 79 (HUMO); R. Vallejo 8 (HUMO); J. Vázquez S. 3712 (HUMO). Int., m., f., r., o. Cult.

36. Cynodon nlemfuensis Vanderyst - N. Espino V. 18 (HUMO); O. López A. 9 (HUMO); J. Maldonado 29 (HUMO); J. Morales O. 145 a (HUMO). Int., m., f., r., o. Cult.

37. Dactyloctenium aegyptium (L.) Willd. - J.R. Dixon 53 (HUMO); C. Feddema 1619 (HUMO); A.S. Hitchcock 6822 (US); O. López A. 64 (HUMO); A. Miranda S. 1189 
Apéndice 2. Continuación.

(HUMO); A. Miranda S. \& P. Guerrero 395 (HUMO); J. Morales O. 93 (HUMO); O. Rodríguez T. s.n. (HUMO). Int., m., f., r., o. Cult.

38. Dinebra aquatica (Scribn. \& Merr.) P.M. Peterson \& N. Snow - A.S. Hitchcock 674 (MO); M. Núñez M. 124 (HUMO); A.M. Prieto S. \& C. Díaz P. s.n. (UAMX); C.G. Pringle 6664 (B, MO, NY, US); M.L. Rodríguez de J. \& C. Díaz P. s.n. (UAMX); V.Q.P.L. \& C. Díaz P. s.n. (UAMX). Nat., r., s.

39. Dinebra panicea (Retz.) P.M. Peterson \& N. Snow subsp. brachiata (Steud.) P.M. Peterson \& N. Snow - A. Aguilar V. et al. s.n. (UAMX); M.L. Aranda s.n. (HUMO); E. Barrios S. \& C. Díaz P. s.n. (UAMX); Blanco O. 15086 (HUMO); L. Campuzano \& C. Díaz P. s.n. (UAMX); I. Castro L. et al. s.n. (UAMX); W. Celis C. \& C. Díaz P. s.n. (UAMX); M.A.D. Díaz A. \& C. Díaz P. s.n. (UAMX); C. Díaz P. \& S. Gómez H. s.n. (UAMX); C. Díaz P. et al. 88 (UAMX); M. García G. \& C. Díaz P. s.n. (UAMX); S. Gaytán O. et al. s.n. (UAMX); M. Gómez C. \& C. Díaz P. s.n. (UAMX); A. Ledezma \& C. Díaz P. s.n. (UAMX); E. Lyonnet 2617 (US); M.R.N.A. \& C. Díaz P. s.n. (UAMX); J. Maldonado 32 (HUMO); E. Medina G. et al. s.n. (UAMX); A. Miranda S. 1204 (HUMO); A. Miranda S. \& P. Guerrero 385 (MO); J. Morales O. 89 (HUMO); C.R. Orcutt 4371 (US); A. Pineda I. \& C. Díaz P. s.n. (UAMX); R. Rodríguez G. \& C. Díaz P. s.n. (UAMX); D. Rodríguez P. \& C. Díaz P. s.n. (UAMX); E. Tovar et al. s.n. (UAMX). Nat., r.

40. Dinebra panicoides (J. Presl.) P.M. Peterson \& N. Snow - M. Núñez M. 95 (HUMO); F. Miranda 1272 (HUMO). Nat., r., s.

41. Dinebra viscida (Scribn.) P.M. Peterson \& N. Snow - F. Miranda 1272 (HUMO). Nat., r., s.

42. Diplachne fusca (L.) P. Beauv. ex Roem. \& Schult. - I. Castro. L. \& C. Díaz P. s.n. (UAMIZ); C. Díaz P. s.n. (UAMIZ); A. Garcéz G. \& C. Díaz P. s.n. (UAMIZ); M. García G. \& C. Díaz P. s.n. (UAMIZ); S. Gaytán O. et al. s.n. (UAMIZ); R. Martínez et al. s.n. (UAMIZ); R.I. Pineda A. \& C. Díaz P. s.n. (UAMIZ); C. Reyes M. \& C. Díaz P. s.n. (UAMIZ); G. Quiñónez L. \& C. Díaz P. s.n. (UAMIZ); J. Vázquez S. 2446 (HUMO). Nat., mp., r.

42a. Diplachne fusca (L.) P. Beauv. ex Roem. \& Schult. subsp. fascicularis (Lam.) P.M. Peterson \& N. Snow - C.G. Pringle 9595 (MO, US). Nat., mp., r.

42b. Diplachne fusca (L.) P. Beauv. ex Roem. \& Schult. subsp. univervia (J. Presl) P.M. Peterson \& N. Snow - O. López A. 45 (HUMO). Nat., mp., r.

43. Disakisperma dubium (Kunth) P.M. Peterson \& N. Snow - R. Alonso S. 4, s.n. (HUMO); A.S. Hitchcock 6851 (US). Nat., f., r. Cult.

44. Distichlis spicata (L.) Greene - G. Ayala A. 37 (MEXU). Nat., ce., r.

45. Eleusine indica (L.) Gaertn. - S. Cacho 23 (HUMO); J.R. Dixon 51 (HUMO); N. Espino V. 21 (HUMO); G. Gándara 22 (HUMO); M. Gutiérrez P. 445 (HUMO); J.C. Juárez D. \& A. Ramírez 331 (HUMO); J.A. León 10 (HUMO); O. López A. 50, 69, 73, 92, s.n. (HUMO); E. Lyonnet 2618 (MO); C.J. Lyons Jr. 56 (US); E. Matuda s.n. (HUMO); A. Miranda S. \& P. Guerrero 340, 358 (HUMO); J.A. Montaño 8 (HUMO); J. Morales O. 87, 99, 111, 124, 
Apéndice 2. Continuación.

148, s.n. (HUMO); J. Morales O. \& López 19, s.n. (HUMO); M. Núñez M. 19, 28, 51, 93, 97, s.n. (HUMO); V. Peñaloza G. 735 (HUMO); M. Reyes B. 1138, 1306 (HUMO); J. Rosado 113 (HUMO); J. Sánchez B. PXXIIA (MEXU); L. Sánchez s.n. (HUMO); A. de los Santos 22 (HUMO); J. Vázquez S. 3882 (HUMO). Int., f., m., o., r. Cult.

46. Enneapogon desvauxii P. Beauv. - R.A. Hernández C. et al. 584 (HUMO, UAMIZ). Nat., f., r., s.

47. Eragrostis capillaris (L.) Nees - G. Andrade \& C.H. Ramos s.n. (HUMO). Nat., r.

48. Eragrostis cilianensis (All.) Vignolo ex Janch. - M.C. Avella M. 60 (HUMO); C.H. Human 92 (HUMO); O. López A. 56 (HUMO); E. Lyonnet 2634 (US); F. Miranda 1279 (HUMO); J. Morales O. 90, 130 (HUMO); M. Núñez M. 49 (HUMO); J.R. Rice 45 (HUMO); O. Rodríguez T. s.n. (HUMO); J.N. Rose et al. 8570 (US). Int., o., mn., r.

49. Eragrostis ciliaris (L.) R.Br. - Alcazar s.n. (HUMO); E. Estrada L. 1329 (MEXU, UAMIZ); O. López A. 101 (HUMO); E. Lyonnet 2612 (US); E. Manrique A.A. Beetle, et al. 729 (HUMO); A. Miranda S. \& P. Guerrero 375 (HUMO); P. Municipal s.n. (HUMO); R.V. Ortega et al. 1577 (HUMO); C.G. Pringle 11223 (MO, US). Nat., f., r. Cult.

50. Eragrostis intermedia Hitchc. - E. Lyonnet 2620 (US); A. Miranda S. \& P. Guerrero 338, 346, 404 (HUMO); M. Reyes B. 1173 (MEXU, UAMIZ). Nat., r.

51. Eragrostis japonica (Walter) L.H. Dewey - Daumás 3 (UAMIZ); G. Gándara s.n. (HUMO); E. Martínez S. et al. 33230 (HUMO, MO); A. Miranda S. \& P. Guerrero 371 (HUMO); J. Morales O. \& López 66 (HUMO); C.G. Pringle 6605 (MO, US); J. Vázquez S. 1740 (HUMO). Int., m., o., r.

52. Eragrostis lugens Nees - A.W. Jackson 63 (HUMO); E. Lyonnet 63 (HUMO); C.H. Thomas 63 (HUMO). Nat., r., s.

53. Eragrostis maypurensis (Kunth) Steud. - M.C. Avella M. 135 (HUMO). Nat., r., s.

54. Eragrostis mexicana (Hornem.) Link - G. Andrade \& C.H. Ramos s.n. (HUMO); G.L. Atmar 96 (HUMO); M.A. Ávila s.n. (HUMO); G. Ayala A. 58, 84 (HUMO); C.E. Boyd 46 (HUMO); J.T. Camp 49 (HUMO); N. Espino V. 17 (HUMO); J.L. Espitia 3 (UAMIZ); O. Gallardo C. s.n. (HUMO); F. Gallegos H. 665 (HUMO); F. González M. \& R. Dirzo 19 (HUMO); J.A. Hernández Q. \& C. Díaz P. s.n. (UAMX); A.S. Hitchcock 6857, 6866 (US); O. López A. 2a, 29 (HUMO); E. Lyonnet 2635 (US); J. Morales O. 94, 118, 132 (HUMO); H. Nolasco N. \& C. Díaz P. s.n. (UAMX); M. Núñez M. 5296 (HUMO); C.R. Orcutt 4373 (HUMO); A. Pulido E. et al. 41 (HUMO); J.T. Villamil P. s.n. (HUMO); G. Woronow \& S. Juzepczuk 909 (US). Nat., r., s.

55a. Eragrostis pectinacea (Michx.) Nees var. miserrima (E. Fourn.) Reeder - A.A. Beetle et al. 1648 (MO); J.A. Chávez P. \& C. Díaz P. s.n. (UAMX); C. Feddema 1615 (UAMX); R. Granados A. \& C. Díaz P. s.n. (UAMX); M. Gutiérrez P. 443 (HUMO); I. Navarro \& C. Díaz P. s.n. (UAMX); C.R. Orcutt 3884 (MO, US); M. Pucheta D. \& C. Díaz P. s.n. (UAMX); J. Vázquez S. 2630 (HUMO). Nat., mp., r., s.

55b. Eragrostis pectinacea (Michx.) Nees var. pectinacea - L.H. Harvey 8670 (MO, US); A.S. Hitchcock 6825, 6867 (US); G. Woronow \& S. Juzepczuk 908 (US), Nat., mp., r., s. 
Apéndice 2. Continuación.

56. Eragrostis pilosa (L.) P. Beauv. - A. Castañeda C. \& C. Díaz P. s.n. (UAMX); H.J. Cortés B. \& C. Díaz P. s.n. (UAMX); T. Hunt 116 (HUMO); V.M. López G. \& C. Díaz P. s.n. (UAMX); E. Manrique et al. 726, 730 (HUMO); E. Martínez A. \& C. Díaz P. s.n. (UAMX). Int., mp., r., s.

57. Eragrostis viscosa (Retz.) Trin. - E. Lyonnet 2795 (US). Int., r.

58. Gouinia virgata (J. Presl) Scribn. - E. Lyonnet 2659 (MO, US). Nat., r., s.

59. Hilaria belangeri (Steud.) Nash - O. López A. 51 (HUMO); J.L. Monsiváis M. s.n. (HUMO); M. Núñez M. 15 (HUMO). Nat., f., r. Cult.

60. Hilaria cenchroides Kunth - M.C. Avella M. 4 (HUMO); J. Morales O. 122, 183 (HUMO); A.J. Sharp 441358 (HUMO). Nat., f., r.

61. Leptochloa virgata (L.) P. Beauv. - J.L. Espitia 4 (HUMO); F.J. Gómez H. s.n. (HUMO). Nat., r.

62. Microchloa kunthii Desv. - C.E. Boyd 42 (HUMO); J.T. Camp 38 (US); Y. Carbajal R. \& L. Galicia G. s.n. (UAMX); R. Cerros T. 123, 1595 (UAMIZ); M. Espinoza M. \& C. Díaz P. s.n. (UAMX); W. Fosberg 74 (HUMO); R.A. Hernández C. et al. 519 (HUMO, UAMIZ); A.S. Hitchcock 6843 (US); M. Núñez M. 10 (HUMO); G. Villegas 1624 (HUMO). Nat., r., s.

63. Muhlenbergia alamosae Vasey - C.G. Pringle 6994 (MO). Nat., r.

64. Muhlenbergia capillaris (Lam.) Trin. - M.S. Antipovitch 69 (MO). Nat., r., o.

65. Muhlenbergia cenchroides (Humb. \& Bonpl. ex Willd.) P.M. Peterson - G. Ayala A. 10, 63, 81 (HUMO); S. Block M. 933 (MEXU); A. Bonfil 401 (UAMIZ); L. Cardoso V. 1369 (IEB, UAMIZ); L. Cardoso V. \& T. Mundo O. 9108 (HUMO); O. López A. 65 (HUMO); E. Lyonnet 2514 (HUMO); E. Lyonnet 626 (HUMO); J. Morales O. 155, 162 (HUMO); J. Morales O. \& López 61 (HUMO); M. Núñez M. 74, 81 (HUMO). Nat., r.

66. Muhlenbergia ciliata (Kunth) Trin. - R.A. Hernández C. et al. 551 (HUMO, UAMIZ); M. Núñez M. 24 (HUMO); C.G. Pringle 11229 (MO). Nat., r.

67. Muhlenbergia diversiglumis Trin. - S. Block M. 35 (MEXU); R.A. Hernández C. et al. 103, 548, 596 (HUMO, UAMIZ); C.G. Pringle 8364, 11234 (HUMO). Nat., r.

68. Muhlenbergia dubia E. Fourn. - G. Ayala A. 45 (HUMO). Nat., r.

69. Muhlenbergia emersleyi Vasey - E. Lyonnet 2873 (HUMO); F. Vázquez S. s.n. (HUMO). Nat., o., r.

70. Muhlenbergia geminiflora (Kunth) P.M. Peterson - S. Block M. 579, 602, 1198 (MEXU); A. Brito 180 (HUMO); R. Cerros T. 1605 (UAMIZ); J. Espinosa 259 (HUMO); R.A. Hernández C. et al. 64, 546, 581 (HUMO, UAMIZ); M. Ishiki 757 (HUMO); O. López A. 30, 33, 74, 84 (HUMO); J.J. Ortiz 1133 (HUMO); F. Sánchez E. s.n. (HUMO); M. Sánchez \& R. Monsalvo s.n. (HUMO); D. Stein 33 (UAMIZ); J. Vázquez S. s.n. (HUMO). Nat., r.

71. Muhlenbergia implicata (Kunth) Trin. - G. Ayala A. 43 (HUMO); A. Brito 181 (HUMO); R. Cerros T. 1601 (UAMIZ); R.A. Hernández C. et al. 770 (HUMO, UAMIZ); M. Núñez M. 44 (HUMO); P. Municipal s.n. (HUMO); J. Sánchez B. PVIA (MEXU); J. Vázquez S. 2626 (HUMO). Nat., r. 
Apéndice 2. Continuación.

72. Muhlenbergia macroura (Kunth) Hitchc. - G. Ayala A. 31 (HUMO); G. Ayala A. 40, 49, s.n. (HUMO); I. Díaz V. 1155 (HUMO); I. Escamilla G. 24, 26, 28 (HUMO); G.L. Fisher s.n. (MO); S. Gaytán O. \& C. Díaz P. s.n. (UAMX); I. Hernández R. \& C. Díaz P. s.n. (UAMX); J. López \& C. Díaz P. s.n. (UAMX); E. Lyonnet 56 (MO), 1408, s.n. (HUMO); E. Manrique et al. 738 (HUMO); J. Morales O. 151 (HUMO); M. Núñez M. 43 (HUMO); J.J. Ortiz \& T. Mejía S. 1128 (MO); A. Pulido E. et al. 23, 55 (UAMIZ). Nat., ce., o., r. Cult.

73. Muhlenbergia microsperma (DC.) Trin. - G. Ayala A. 60, 75, 76, 86 (HUMO). Nat., r., s.

74. Muhlenbergia minutissima (Steud.) Swallen - G. Ayala A. 65 b (HUMO); E. Lyonnet 1901 (HUMO); J. Morales O. 167 (HUMO). Nat., r., s.

75. Muhlenbergia mutica (Rupr ex E. Fourn.) Hitchc. - E. Lyonnet 2872 (HUMO). Nat., r., s.

76. Muhlenbergia nigra Hitchc. - I. Escamilla G. s.n. (HUMO). Nat., ce., o., r.

77. Muhlenbergia orophila Swallen - E. Matuda 25599, 25601 (MO, US). Nat., r., s.

78. Muhlenbergia pectinata C.O. Goodding - A.S. Hitchcock 1355 (GH, MO, NY). Nat., r., s.

79. Muhlenbergia pereilema P.M. Peterson - E. Lyonnet 1353 (US), s.n. (MO); C.G. Pringle 11245 (MO, US); 11764 (MO). Nat., r., s.

80. Muhlenbergia peruviana (P. Beauv.) Steud. - G. Ayala A. 65a (HUMO); C.G. Pringle 11743 (MO). Nat., r., s.

81. Muhlenbergia phalaroides (Kunth) P.M. Peterson - I. Díaz V. 1100 (HUMO); W. Fosberg 78 (HUMO, US). Nat., f., r.

82. Muhlenbergia phleoides (Kunth) Columbus - M.C. Barrera R. \& C. Díaz P. s.n. (UAMX); C.E. Boyd 51 (HUMO); J.T. Camp 43 (US); M.A. Flores V. \& C. Díaz P. s.n. (UAMX); O. López A. 31 (HUMO); R. Martínez S. 96 (HUMO); F. Mendoza D. \& C. Mercado s.n. (UAMX); M. Núñez M. 41 (HUMO); F. Robles P. \& C. Díaz P. s.n. (UAMX). Nat., f., r.

83. Muhlenbergia plumiseta Columbus - W. Boege 1973 (HUMO); E. Lyonnet 2668 (MO, US); C.G. Pringle 5962, 11243 (MO). Nat., r., s.

84. Muhlenbergia quadridentata (Kunth) Trin. - E. Lyonnet 2496 (HUMO); J. Morales O. 168 (HUMO). Nat., r., s.

85. Muhlenbergia rigida (Kunth) Kunth - L. Cardoso V. 1375 (HUMO); R.A. Hernández C. et al. 30 (HUMO, UAMIZ); R. Martínez S. 115 (HUMO); A. Miranda S. \& P. Guerrero 411 (HUMO); M. Núñez M. 13 (HUMO). Nat., r., s.

86. Muhlenbergia robusta (E. Fourn.) Hitchc. - G. Ayala A. 6 (HUMO); S. Block M. 1291 (MEXU); E. Estrada L. 1867 (UAMIZ); L. Hahn 420 (MO); R.A. Hernández C. R. Cerros T., A. Flores M., E. González R., M. Vázquez M. 26, 587, 623 (HUMO, UAMIZ); E. Lyonnet 2553 (MO); J. Morales O. 192 (HUMO); P. Municipal s.n. (HUMO); A. Pulido E. et al. 29 (UAMIZ); J.F. Zúñiga P. s.n. (HUMO). Nat., r., s.

87. Muhlenbergia tenella (Kunth) Trin. - A. Brito 182 (HUMO); R. Cerros T. 1608 (UAMIZ); G. Flores 157 (HUMO); R.A. Hernández C. et al. 76 (HUMO, UAMIZ); 
Apéndice 2. Continuación.

E. Lyonnet 2669 (MO); E. Matuda s.n. (HUMO); A. Miranda S. 336 (HUMO); P. Municipal s.n. (HUMO); C.G. Pringle 11232 (MO); B. Torres 271 (HUMO). Nat., r., s.

88. Muhlenbergia tricholepsis (Torr.) Columbus - E. Lyonnet 45 (MO), s.n. (HUMO). Nat., r., s.

89. Muhlenbergia versicolor Swallen - S. Block M. 1325 (MEXU); R.A. Hernández C. et al. 27 (HUMO, UAMIZ); E. Lyonnet 1838 (HUMO, MO); M. Núñez M. 84 (HUMO). Nat., r., s.

90. Muhlenbergia virletii (E. Fourn.) Soderstr. - S. Block M. 1015 (MEXU). Nat., r., s.

91. Pappophorum mucronulatum Nees - M. Quezada R. 1622 (HUMO). Nat., r.

92. Sporobolus indicus (L.) R.Br. - G. Ayala A. 33, 66, 73, 89 (HUMO); A. Brito 77 (HUMO); J.T. Camp 50 (HUMO); F. Gallegos H. 659 (HUMO); M. Gutiérrez P. 405 (HUMO); J.T. Harris 71 (US); A.S. Hitchcock 68, 6827 (US); O. López A. 14, 83 (HUMO); E. Lyonnet \& J. Elcoro 1810 (MEXU, US); D. Martínez A. s.n. (HUMO); E. Matuda 439 (HUMO); A. Miranda S. \& P. Guerrero 365 (HUMO); J. Morales O. 68, 115, 156 (HUMO); M. Núñez M. 20, 79 (HUMO); C.R. Orcutt 2375 (HUMO); J. Rosasco 125 (HUMO); G. Woronow \& S. Juzepczuk 885 (US). Nat., r., s.

93. Sporobolus trichodes Hitchc. - R.A. Hernández C. et al. 556 (HUMO, UAMIZ); J. Sánchez B. PXVIA (MEXU). Nat., r., s.

94. Tetrapogon chlorideus (J. Presl) P.M. Peterson - C. Díaz P. et al. 107, 118 (UAMX); F. Jiménez C. \& C. Díaz P. s.n. (UAMX); J. Morales O. \& López 13 (HUMO); H. Nolasco N. \& C. Díaz P. s.n. (UAMX); C.G. Pringle 1902 (MO, US); Y. Santiago G. \& C. Díaz P. s.n. (UAMX); M.E. Vela G. \& C. Díaz P. 37, 38 (UAMX). Nat., r., s.

Danthonioideae H.P. Linder \& N.P. Barker

95. Cortaderia selloana (Schult.) Asch. \& Graebn. - J.G. Sánchez-Ken \& J.I. Calzada 656 (ISC). Int., ce., o., r. Cult.

Ehrhartoideae Jacq.-Fél. ex Caro

96. Leersia hexandra Sw. - N. Espino V. 20 (MEXU); M. García G. s.n. (UAMX); A.S. Hitchcock 6847 (US); M. Núñez M. 123 (MEXU). Nat., f., mp. Cult.

97. Oryza sativa L. - E. Aguirre 8336 (MEXU); W. Celis C. \& C. Díaz P. s.n. (UAMX); T. García M. \& C. Díaz P. s.n. (UAMX); A. González \& B. Rodríguez s.n. (UAMIZ); A. Hernández G. \& C. Díaz P. s.n. (UAMX); A. Huchin G. \& C. Díaz P. s.n. (UAMX); J. Maldonado 13 (UAMIZ); E. Matuda s.n. (MEXU); A. Miranda S. \& P. Guerrero 360 (MEXU); M. Núñez M. 91, 126 (MEXU); I. Rivera 5 (MEXU); J. Viricio C. s.n. (MEXU). Int., a., f., mn., r. Cult.

Panicoideae A. Braun

98. Andropogon fastigiatus Sw. - R. Cerros T. et al. 2619, 2730, 2732, 2733 (HUMO, MEXU); E. Lyonnet 2810 (US); C.G. Pringle 11211 (MEXU, MO); J. Sánchez B. PXXVIIA (MEXU); G. Woronow \& S. Juzepczuk 880 (US). Nat., r.

99. Andropogon gayanus Kunth - M. Quezada R. 1869 (MEXU). Int., f., r. Cult.

100. Andropogon glomeratus (Walter) Britton, Sterns \& Ponggeb. - G. Gándara 1912 (MEXU); E. Lyonnet 2890 (US). Nat., o., s., mp., me. 
Apéndice 2. Continuación.

101. Anthephora hermaphrodita (L.) Kuntze - R. Cerros T. 1163 (MEXU); M.S. Macías L. s.n. (UAMX); O. Martínez R. s.n. (UAMX); A. Miranda S. 1194 (MEXU). Nat., s., ce., op.

102. Arundinella berteroniana (Schult.) Hitchc. \& Chase - E. Lyonnet 3170 (MEXU); E. Matuda 26012, 26013, 26017 (MO). Nat., r.

103. Arundinella deppeana Nees - E. Matuda 25986 (MO); A. Miranda S. \& P. Guerrero 354 (MEXU). Nat., r.

104. Axonopus aff. compressus (Sw.) P. Beauv. - J. Vázquez S. 3487 (MEXU). Nat., r., o. Cult.

105. Bothriochloa alta (Hitchc.) Henrard - R. Guzmán M. 4313 (MEXU). Nat., r., s.

106. Bothriochloa barbinodis (Lag.) Herter - R. Cerros T. 1616 (UAMIZ); R. Cerros T. al. 2644 (HUMO, MEXU); J. Maldonado 33 (HUMO). Nat., r., ce., o., f.

107. Bothriochloa hirtifolia (J. Presl) Henrard - C.G. Pringle 8702 (MEXU, MO, TAES). Nat., r., ce., o.

108. Bothriochloa ischaemum (L.) Keng - R. Cerros T. et al. 2682 (HUMO, MEXU); J. Rosasco 122 (MEXU). Int., f., mp. Cult.

109. Bothriochloa laguroides (DC.) Herter - C.E. Boyd 45 (MEXU); J.T. Camp 40 (MEXU); E. Matuda 26084 (MEXU, MO); J.N. Rose 10194 (US); C.H. Thomas 58 (MEXU). Nat., r., ce., o., f.

110. Bothriochloa longipaniculata (Gould) Allred \& Gould - F. González s.n. (UAMX); E. Jacinto s.n. (UAMX); O. López A. 42 (MEXU). Nat., r., ce., o., f.

111. Bothriochloa perforata (Trin. ex E. Fourn.) Herter - M. Núñez M. 37 (MEXU). Nat., r., ce., o., f.

112. Bothriochloa pertusa (L.) A. Camus - R. Cerros T. et al. 2674 (HUMO); G. Velázquez O. s.n. (UAMX). Int., r., ce., op., f. Cult.

113. Bothriochloa wrightii (Hack.) Henrard - R. Cerros T. 1611 (UAMIZ); E. Lyonnet 1861 (MEXU); A. Miranda S. 409 (MEXU). Nat., ce., o., r.

114. Cenchrus brownii Roem. \& Schult. - N. Hernández C. s.n. (UAMX); F. Ortiz F. s.n. (UAMX). Nat., s., r.

115. Cenchrus ciliaris L. - S. Aguirre y C. 158 (MEXU); R. Alonso S. 8, s.n. (HUMO); J.G. Ariza B. 2, s.n. (HUMO); M.C. Avella M. 75 (MEXU); N. Becerra M. s.n. (MO); R. Cerros T. et al. 2651, 2727 (HUMO, MEXU); O. Domínguez S. 99 (MEXU); E. Estrada L. 1163 (MEXU, UAMIZ); E.H. Gómez G. s.n. (UAMX); R.A. Hernández C. et al. 582 (HUMO, UAMIZ); A.W. Lievens \& B.M. Gregory, Jr. 3026 (MEXU); O. Martínez R. s.n. (UAMX); A. Miranda S. 1221 (MEXU); J. Morales O. López 78 (MEXU); J. Olguín P. s.n. (UAMX); L. Pacheco 59 (MEXU); C. Ronzo R. s.n. (UAMX); S. Zárate 491 (MEXU). Intr., mn. Cult.

116. Cenchrus clandestinus (Hochst. ex Chiov.) Morrone - J. Bonilla \& C.T. Philbrick 1325 (HUMO, MEXU); G. Villegas 1625 (MEXU). Int., mn., o., r. Cult.

117. Cenchrus echinatus L. - G. Andrade \& C.H. Ramos s.n. (MEXU); G.L. Atmar 66 (MEXU); M.C. Avella M. 21 (MEXU); Bilimek 447 (US); E. Cabrera C. \& G. Flores 
Apéndice 2. Continuación.

12211 (MEXU); R. Cerros T. et al. 2683, 2726 (HUMO, MEXU); E. Estrada L. 1190 (MEXU); F. Gallegos H. et al. 443 (MEXU); R. Granados A. s.n. (UAMX); M. Gutiérrez P. 501 (MEXU); A.S. Hitchcock 6852, 6876 (US); A.W. Jackson 64 (MEXU); J. Lobato s.n. (UAMIZ); C.J. Lyons Jr. 116 (MEXU, US); E. Manrique et al. 728, 731 (MEXU); G. Martínez S. s.n. (UAMX); F. Miranda 1669 (MEXU); A. Miranda S. \& P. Guerrero 361 (MEXU), 389 (MEXU, MO); J. Morales O. López 1, $96 a$ (MEXU); J.L. Paz J. s.n. (UAMX); T.R. Soderstrom 504 (MEXU, US); D. Vargas C. s.n. (UAMX). Nat., mn., r.

118. Cenchrus michoacanus (Kunth) Morrone - F. Domínguez R. 104, 315 (MEXU); J.A. León 8 (MEXU). Nat., mp., r.

119. Cenchrus pilosus Kunth - A. Bonfil 278, 387 (UAMIZ); G. García M. s.n. (UAMX); E. Lyonnet 2622 (US); C.J. Lyons Jr. 85 (US); A. Miranda S. 1188 (MEXU); C.G. Pringle 11219 (MO, US); M. Reyes B. 1300 (UAMIZ); D. Stein 41 (HUMO). Nat., mn., r.

120. Cenchrus polystachios (L.) Morrone - C.G. Pringle 11241 (MO, US); J. Vázquez S. 1104, 2313, 3952 (MEXU). Int., m., r.

121. Cenchrus purpureus (Schumach.) Morrone - R. Cerros T. et al. 2697 (HUMO, MEXU); J. Vázquez S. 1577 (MEXU). Int., f., m., r. Cult.

122. Cenchrus setaceus (Forssk.) Morrone - R. Cerros T. et al. 2521 (HUMO, MEXU); $R$. Guzmán M. 5931 (MEXU). Int., mp., o., r. Cult.

123. Cenchrus spinifex Cav. - G. Gándara s.n. (MEXU); A. Sotelo G. et al. 3336 (HUMO). Nat., mn., r.

124. Cenchrus villosus (Spreng.) Spreng. - R. Medina 41 (UAMIZ), s.n. (HUMO); J. Vázquez S. 1316 (MEXU). Int., mp., o., r. Cult.

125. Chaetium bromoides (J. Presl) Benth. ex Hemsl. - A. Díaz O. 19 (MEXU); J. García P. 1716 (MEXU); A. González \& B. Rodríguez s.n. (MEXU); O. López A. 82, 86 (MEXU); E. Lyonnet 2447 (MEXU); E. Matuda 25920 (MEXU); J. Morales O. 191 (MEXU). Nat., r., s.

126. Coix lacryma-jobi L. - M. Saint-Pierre 907 (US); B. Torres 244 (MEXU). Int., o., u. Cult.

127. Cymbopogon citratus (DC.) Stapf - González-Barrales (2006). Int., o., me. Cult.

128. Dichanthelium commutatum (Schult.) Gould - R. Cerros T. 2543 (HUMO, MEXU); $O$. López A. 80 (MEXU); E. Lyonnet 623 (MEXU, MO); M. Núñez M. 68 (MEXU). Nat., r., s.

129. Dichanthium annulatum (Forssk.) Stapf - J.A. Chávez P. s.n. (UAMX); R.H. Díaz C. s.n. (UAMX); J.R. Gaytán M. s.n. (UAMX). Int., r.

130. Digitaria argillacea (Hitchc. \& Chase) Fernald - R. Cerros T. et al. 2579, 2580 (HUMO, MEXU); A.S. Hitchcock 6828 (US), 6864 (MEXU, US); E. Lyonnet 2809 (MEXU, US); C.J. Lyons Jr. 82 (MEXU, US). Nat., r., s.

131. Digitaria badia (Scribn. \& Merr.) Fernald - E. Lyonnet 2429a, 2442a (MEXU); sin colector s.n. (MEXU). Nat., r., s.

132. Digitaria bicornis (Lam.) Roem. \& Schult. - R. Cerros T. 2507 (HUMO, MEXU); A. Huchin G. s.n. (UAMX); R. Meléndez C. s.n. (UAMX); A. Miranda S. \& P. 
Apéndice 2. Continuación.

Guerrero 356 (MEXU); J. Morales O. 86, 133 (MEXU); J. Moreno de la H. s.n. (UAMX). Int., $r$.

133. Digitaria ciliaris (Retz.) Koeler - R. Cerros T. et al. 2554b, 2601, 2655 (HUMO, MEXU); R. Guzmán M. 4310 (MEXU); A.S. Hitchcock 6829 (US); O. López A. 58, 71, 93 (MEXU); E. Lyonnet $2809 a$ (MEXU); C.J. Lyons Jr. 105a (US); A. Miranda S. \& P. Guerrero 337, 384 (MEXU); J. Morales O. 107, 159, 184 (MEXU); J. Morales O. \& López 23 (MEXU); M. Núñez M. 103, 184 (MEXU); D. Pipes 74 (US); sin colector s. $n$. (MEXU); R.D. Webster 89 (US), 1989 (MO). Nat., ce., f., mp., r.

134. Digitaria filiformis (L.) Koeler var. laeviglumis (Fernald) Wipff - J.F. Suárez F. s.n. (UAMX). Nat., r.

135. Digitaria horizontalis Willd. - J.C. Juárez D. et al. 260 (MEXU). Nat., r.

136. Digitaria insularis (L.) Fedde - F. Gallegos H. 442 (MEXU); A.S. Hitchcock 6858 (US); O. López A. 99 (MEXU); E. Matuda 26007, 26078 (MEXU); M. Quezada R. 1958 (MEXU). Nat., op., r., s.

137. Digitaria ischaemum (Schreb.) Muhl. - R.A. Hernández C. et al. 577 (HUMO, UAMIZ). Int., m.

138. Digitaria leucites (Trin.) Henrard - R. Cerros T. et al. 2709 (HUMO, MEXU); F. Gallegos H. 499 (MEXU); O. López A. 15, 81a (MEXU); E. Lyonnet 58, 1879 (MEXU, MO), 2429 (MEXU, US), 2442, 2540 (MEXU); A. Miranda S. \& P. Guerrero 410 (MEXU); F. Morales J. s.n. (UAMX); M. Núñez M. 71 (MEXU); P. Tenorio L. 18977 (MEXU). Nat., op., r., s.

139. Digitaria milanjiana (Rendle) Stapf - R. Cerros T. et al. 2649 (HUMO, MEXU). Int., f., r. Cult.

140. Digitaria nuda Schumach. - A.W. Jackson 40 (MEXU); S. Vázquez E. s.n. (UAMX). Int., f., r.

141. Digitaria sanguinalis (L.) Scop. - M. González \& C. Díaz P. s.n. (UAMX). Int., f., r., mp. Cult.

142. Digitaria sp.- D. García P. s.n. (UAMX); O. López A. 62 (MEXU); Y. Medellín C. s.n. (UAMX). Nat., op., r., s.

143. Digitaria ternata (Hocht. ex A. Rich.) Stapf - R. Cerros T. et al. 2560, 2628 (HUMO, MEXU); U. González Q. 642 (MEXU); O. López A. 3, 16 (MEXU); J. Morales O. 98, 119 (MEXU); J. Morales O. \& López 24 (MEXU); M. Núñez M. 8, 22, 29 (MEXU); J. Sánchez B. PXXVIA (MEXU). Int., r.

144. Digitaria velutina (Forssk.) P. Beauv. - J. Alanís C. s.n. (UAMX); F.E. Esquivel R. s.n. (UAMX); D. González Z. s.n. (UAMX). Int., m.

145. Echinochloa colona (L.) Link - R. Alonso S. 2, s.n. (HUMO); A. Bonfil 107, s.n. (HUMO); S. Cacho 80, s.n. (HUMO); R. Cerros T. et al. 2530, 2605, 2653 (HUMO, MEXU); E. Cervantes 7 (HUMO); R.I. Hernández A. \& J.L. Mendoza G. s.n. (UAMX); M.J. Hernández F. s.n. (UAMX); S. Hernández G. s.n. (UAMX); A.S. Hitchcock 6836 (US); J. Maldonado 27a (HUMO); M.A. Mijangos C. 41 (MEXU); A. Sandoval F. s.n. (UAMX); D. Stein s.n. (HUMO). Nat., f., mp., r. 
Apéndice 2. Continuación.

146. Echinochloa crus-galli (L.) P. Beauv. - T. Flores et al. s.n. (UAMX); A.S. Hitchcock 6849 (US); S.D. Koch 75315 (US); L.M. Velasco S. \& M.N. Fernández s.n. (UAMX). Nat., f., mp., r.

147. Echinochloa crus-pavonis (Kunth) Schult. - E. Martínez A. s.n. (UAMX); A. Soberanes s.n. (UAMX); Y. Toledo M. s.n. (UAMX). Nat., r., f.

148. Echinochloa holciformis (Kunth) Schult. - G. Gándara 5 (US). Nat., f., o., r.

149. Echinochloa oplismenoides (E. Fourn.) Hitchc. - A. Godinez V. s.n. (UAMX). Nat., r.

150. Elionurus ciliaris Kunth - M.S. Antipovitch 63 (MO); R. Cerros T. et al. 2592 (HUMO, MEXU); J. Sánchez B. PXIA (MEXU). Nat., r., s.

151. Elionurus tripsacoides Humb. \& Bonpl. ex Willd. - A.S. Hitchcock 6846 (US); O. López A. 38 (MEXU); A. Miranda S. \& P. Guerrero 353 (MO). Nat., r., s.

152. Eriochloa acuminata (J. Presl) Kunth var. acuminata - M. Almazán V. s.n. (UAMX); N. Becerra M. 39 (MO); R. Cerros T. 2510 (HUMO, MEXU); R. Cerros T. et al. 1354 (MEXU), 2531, 2552, 2650 (HUMO, MEXU); C. Díaz P. s.n. (UAMX); C. Díaz P. \& R. Granados s.n. (UAMX); A.W. Jackson 39 (MEXU); S.D. Koch 75314 (US); O. López A. 48 (MEXU); J. Morales O. 128 (MEXU); J. Morales O. \& López 16 (MEXU); J.T. Oguer s.n. (MEXU); J.L. Paz J. s.n. (UAMX); H. Tenorio L. s.n. (UAMX); J. Vázquez S. 1788, 3410 (MEXU). Nat., r.

153. Eriochloa nelsonii Scribn. \& J.G. Sm. - R. Cerros T. et al. 2680 (HUMO, MEXU); E. Estrada L. 1397 (MEXU); G. Itié s.n. (MEXU); E. Lyonnet 2628 (MEXU, MO, US); R. Méndez L. 239 (MEXU); F. Miranda 1649 (MEXU); C.G. Pringle 11224 (MEXU, MO, US). Nat., r.

154. Eriochloa punctata (L.) Desv. - C.G. Pringle 9605 (US); D. Vargas C. s.n. (UAMX); H. Vibrans 7642 (MEXU). Nat., r.

155. Euclasta condylotricha (Steud.) Stapf - E. Lyonnet 2664 (MEXU, MO, US). Int., r.

156. Hemarthia altissima (Poir.) Stapf \& C.E. Hubb. - C.G. Pringle 9610 (MEXU, US). Int., f., $r$.

157. Heteropogon contortus (L.) P. Beauv. ex Roem. \& Schult. - O. Bruno E. s.n. (UAMX); R. Cerros T. 160 (HUMO), 2514 a (HUMO, MEXU); R. Cerros T. et al. 2574, 2618, 2677 (HUMO, MEXU); A.S. Hitchcock 6871 (US); E. Lyonnet 2637 (MO, US); E. Lyonnet \& J. Elcoro 1174 (US); P.J. Lyons 97 (US); J. Morales O. 174 (MEXU). Int., ce., f., mn., r. Cult.

158. Heteropogon melanocarpus (Elliott) Benth. - W. Celis C. s.n. (UAMX); R. Cerros T. 1612 (UAMIZ); R. Cerros T. et al. 2611 (HUMO, MEXU); J. Galicia X. s.n. (UAMX); A.S. Hitchcock 6877 (US); O. López A. 105 (MEXU); E. Lyonnet 2815 (US); C.G. Pringle 8678 (MO, US); G. Woronow 2415 (US). Int., ce., f., mn., r.

159. Hopia obtusa (Kunth) Zuloaga \& Morrone - S. Alvarez R. s.n. (UAMX); R. Cerros T. et al. 2643 (HUMO, MEXU); C. Díaz P. s.n. (UAMX); M.M. Hernández A. s.n. (UAMX); O. Palafox (UAMX). Nat., s.

160. Hyparrhenia rufa (Nees) Stapf - R. Cerros T. 11a (HUMO, MEXU); M. Núñez M. 105 (MEXU); Y. Toledo M. s.n. (UAMX). Int., mp., r. 
Apéndice 2. Continuación.

161. Hyparrhenia variabilis Stapf - R. Cerros T. et al. 2693 (HUMO, MEXU); J.G. SánchezKen et al. s.n. (IBUG, MEXU, XAL). Int., r., m.

162. Hyperthelia dissoluta (Nees ex Steud.) Clayton - R. Guzmán M. 4316 (MEXU); E. Morales s.n. (UAMX); V.H. Moreno S. s.n. (UAMX). Int., r.

163. Imperata sp. - J.L. Brunhuber - F. Calderón et al s.n. (HUMO). Nat., mn., op., r.,

164. Ixophorus unisetus (J. Presl) Schltdl. - G.L. Atmar 89 (MEXU); N. Becerra M. s.n. (MO); W. Celis C. s.n. (UAMX); R. Cerros T. 13, $2511 b$ (HUMO, MEXU); R. Cerros T. et al. 2533 (HUMO, MEXU); E. Cervantes s.n. (UAMIZ); A.A. Domínguez A. s.n. (UAMX); G. Gándara 3 (US); F. González M. \& R. Dirzo 18 (MEXU); A.S. Hitchcock 6821, 6841 (US); A. Miranda S. \& P. Guerrero 388 (MEXU); J.L. Monsiváis M. 15 (UAMIZ); J. Morales O. 106 (MEXU); J. Morales O. \& López 8, 43 (MEXU); M. Núñez M. 94, 122 (MEXU); C.R. Orcutt 4407 (US); B. Osorio R. s.n. (UAMX); V. Peñaloza G. 743 (MEXU); C.G. Pringle 8493 (MEXU, MO, US); J.R. Reeder \& C.G. Reeder 4398 (ARIZ, US); J. Rosado 117 (MEXU); A. de los Santos 24 (UAMIZ); V. Vázquez T. 659 (MEXU); G. Woronow \& S. Juzepczuk 866 (US). Nat., mp., r.

165. Lasiacis divaricata (L.) Hitchc. - A. Bonfil 174 (UAMIZ); R.G. Serrano J. 10 (UAMIZ). Nat., op., r., s.

166. Lasiacis nigra Davidse - M.S. Antipovitch 102 (MO); S. Block M. 1276 (MEXU); C.E. Boyd 82 (MEXU); A. Brito 176 (MEXU); R. Cerros T. 2512 (HUMO, MEXU); R. Cerros T. et al. 2563 (HUMO, MEXU); J.R. Dixon 77 (MEXU); Q.F. Fuchs 130 (MEXU); A.S. Hitchcock 6824 (US); W.C. Leavenworth \& M.M. Leavenworth 927 (F, MO); O. López A. 63 (MEXU); M. Luna A. \& C. Díaz P. s.n. (UAMX); E. Lyonnet 1373 (MEXU, US), 2541 (MEXU, MO, US), 2827 (US); F. Miranda 1591 (MEXU); H. Paz S. \& C. Díaz P. s.n. (UAMX); C.G. Pringle 5960 (MEXU, US), 6663 (MEXU, MO, US); H. Ross 254 (US); M. Sánchez \& R. Monsalvo s.n. (MEXU); sin colector 4292 (US); J. Vázquez S. 3757 (MEXU); G. Woronow 2460 (US); G. Yatskievych 312 (HUMO); G. Yatskievych \& G.J. Gastony s.n. (HUMO). Nat., op., r., s.

167. Lasiacis procerrima (Hack.) Hitchc. - S. Block M. 1278 (MEXU). Nat., op., r., s.

168. Lasiacis rhizophora (E. Fourn.) Hitchc. - E. Lyonnet 2888 (US). Nat., op., r., s.

169. Lasiacis ruscifolia (Kunth) Hitchc. - Bilimek 449 (US); E. Cabrera C. 12133 (MEXU); E. Cabrera C. \& G. Flores 14721 (MEXU); R. Cerros T. et al. 2684 (HUMO, MEXU), 2737 (MEXU); E. Estrada L. 1422 (HUMO); W.C. Leavenworth \& M.M. Leavenworth 913 (F); E. Lyonnet 2172 (US), 2658 (MO); M.S. Macias L. \& C. Díaz P. s.n. (UAMX); F. Miranda 1654 (MEXU); G. Naranjo \& C. Díaz P. s.n. (UAMX); C.G. Pringle 11293 (MO, US); A. Rosas H. \& C. Díaz P. s.n. (UAMX); E. Severe \& C. Díaz P. s.n. (UAMX); I. Trejo 1088 (MEXU). Nat., op., r., s.

170. Megathyrsus maximus (Jacq.) B.K. Simon \& S.W.L. Jacobs - R. Cerros T. 2514 (HUMO, MEXU); J.A. Chávez P. s.n. (UAMX); E. Estrada L. 1428 (UAMIZ); G. García M. s.n. (UAMX); A. Ibarra s.n. (UAMX); E. Lyonnet 2650 (HUMO); C.J. Lyons Jr. 153 (US); M. Reyes B. 1314 (UAMIZ). Int., f., mp., op., r. Cult.

171. Melinis minutiflora P. Beauv. - J. Vázquez S. 2104 (MEXU). Int., ce., f., mp., op., r. 
Apéndice 2. Continuación.

172. Melinis repens (Willd.) Zizka - S. Block M. 114 (MEXU); A. Bonfil 302 (HUMO); R. Cerros T. 129, 1613 (UAMIZ); E. Estrada L. 1124, 1678, 1725 (HUMO); R.A. Hernández C. et al. 586 (HUMO, UAMIZ); R. Medina 52 (UAMIZ); A. Pulido E. et al. 33, 42 (HUMO). Int., ce., f., mp., op., r.

173. Mnesithea granularis (L.) de Koning \& Sosef - R. Cerros T. et al. 2598, 2614 (HUMO, MEXU); R.H. Díaz C. s.n. (UAMX); O. Domínguez S. 103 (MEXU); M. García G. \& C. Díaz P. s.n. (UAMX); A.S. Hitchcock 6870 (US); R.M. Lagunas M. et al. s.n. (UAMX); O. López A. \& C. Díaz P. s.n. (UAMX); E. Lyonnet 2644 (MO, US); L. Peña O. \& C. Díaz P. s.n. (UAMX); E. Pineda S. \& C. Díaz P. s.n. (UAMX); C.G. Pringle 11228 (MO, US); G. Woronow \& S. Juzepczuk 889 (US). Int., mp., r.

174. Morronea trichidiachnis (Döll) Zuloaga \& Scataglini - R. Cerros T. et al. 2585 (HUMO, MEXU). Nat., op., s.

175. Oplismenus burmannii (Retz.) P. Beauv. - S. Block M. 62, 97, 759 (MEXU); E. Bourgeau 1301 (MO); E. Castrejón V. 1398 (UAMIZ); R. Cerros T. 125, 1604 (UAMIZ); $R$. Cerros T. et al. 2566, 2583 (HUMO, MEXU); R.A. Hernández C. et al. 46, 105, 131 (HUMO, UAMIZ); E. Lyonnet \& J. Elcoro 1802 (MO, US); C.G. Pringle 6209, 11330 (MO, US); J. Sánchez B. PXXVA (MEXU). Nat., r., s.

176. Oplismenus compositus (L.) P. Beauv. - S. Block M. 893, 1373 (MEXU); R. Cerros T. 2539 (HUMO, MEXU); E. Lyonnet 259 (MO, US); E. Lyonnet \& J. Elcoro 1823 (MO, US); G. Yatskievych 313 (UAMIZ). Nat., mp., op., r.

177. Oplismenus hirtellus (L.) P. Beauv. - A. Bonfil 124 (UAMIZ); A.S. Hitchcock 423 (MO, US), 6839 (US); S. Ortiz et al. 3593 (UAMIZ); C.G. Pringle 6203 (MO, US); M. Vargas 25 (UAMIZ). Nat., mp., op., r.

178. Panicum alatum Zuloaga \& Morrone var. minus (Andersson) Zuloaga \& Morrone - $R$. Cerros T. $2500 b$ (HUMO, MEXU); C. Díaz P. s.n. (UAMX); M. Gutiérrez P. 383 (MEXU); A.W. Jackson 15 (MEXU); C.J. Lyons Jr. 59, 83 (MEXU, US); A. Peñafiel E. \& J. Flores A. s.n. (UAMX); J.R. Walther 63 (US). Nat., r., s.

179. Panicum ghiesbreghtii E. Fourn. - F. Gallegos H. 440 (MEXU). Nat., r., s.

180. Panicum hirticaule J. Presl - M.L. Aranda s.n. (US). Nat., a., op., r. Cult.

181. Panicum lepidulum Hitchc. \& Chase - de la Mora 521 (MEXU); J. Vázquez S. 2627, 3592 (MEXU). Nat., r., s.

182. Panicum parcum Hitchc. \& Chase - E. Lyonnet 2639 (MEXU, MO, US). Nat., r., s.

183. Panicum trichoides Sw. - E. Estrada L. 763 (MEXU); E. Lyonnet 2615 (US); L.A. Pérez J. 2098 (MEXU). Nat., s.

184. Paspalum acuminatum Raddi - R.S. Lagunas M. s.n. (UAMX); G. Naranjo s.n. (UAMX). Nat., s., r.

185. Paspalum botterii (E. Fourn.) Chase - R. Cerros T. et al. 2551 (HUMO, MEXU); 2561 (HUMO, MEXU); M. Gutiérrez P. 446 (MEXU); A.S. Hitchcock 6826 (US); E. Lyonnet 2663 (MO, US); C.J. Lyons Jr. 105 (MEXU); F. Miranda 1278 (MEXU); A. Miranda S. \& P. Guerrero 390 (MEXU); L.M. de Puga V. 7276 (IBUG); G.B. Ross s.n. (US); G. Woronow \& S. Juzepczuk 891 (US). Nat., r., s. 
Apéndice 2. Continuación.

186. Paspalum candidum (Humb. \& Bonpl. ex Flüggé) Kunth - E. Lyonnet 1371 (MEXU, MO, US). Nat., s.

187. Paspalum conjugatum P.J. Bergius - J.R. Dixon 54 (MEXU); F. Gallegos H. \& P. Guerrero s.n. (MEXU); A.S. Hitchcock 6818 (US); O. López A. 81 (MEXU); E. Lyonnet 2661 (MEXU, MO, US), 2808 (MEXU); A. Miranda S. \& P. Guerrero 372, 394 (MEXU); M. Núñez M. 119 (MEXU); C.G. Pringle 6215 (MEXU, MO, US). Nat., mp., o., r.

188. Paspalum conspersum Schrad. - J.N. Rose et al. 8555 (US). Nat., r., s.

189. Paspalum convexum Humb. \& Bonpl. ex Flüggé - R. Cerros T. 1598 (UAMIZ); $R$. Cerros T. et al. 2529, 2546, 2556, 2564, 2578, 2599, 2625, 2673 (HUMO, MEXU); $N$. Espino V. 13, 22, 23 (MEXU); R.A. Hernández C. et al. 511 (HUMO, UAMIZ); A.S. Hitchcock 6830, 6863, 6868 (US); O. López A. 32 a 53 (MEXU); E. Lyonnet 2662a (MEXU); A. Miranda S. 1182 (MEXU); A. Miranda S. \& P. Guerrero 342 (MEXU); J. Morales \& O. López 49, s.n. (MEXU); M. Núñez M. 11, 30 (MEXU); C.R. Orcutt 3889 (US); A. Pulido E. et al. 104 (UAMIZ); J. Sánchez B. PXA (MEXU); J. Vázquez S. 2631, 2634 (MEXU). Nat., r., s.

190. Paspalum denticulatum Trin. - R. Cerros T. et al. 2612 (HUMO, MEXU); F. Domínguez R. 313 (IBUG, MEXU); R. Guzmán M. 4307 (IBUG); A.S. Hitchcock 6823, 6880, 6881 (US); O. Morrone et al. 7368 (SI); M. Núñez M. 50 (MEXU); J.R. Reeder \& C.G. Reeder 4397 (ARIZ, US). Nat., r., s.

191. Paspalum dilatatum Poir. - R. Cerros T. 1615 (UAMIZ). Nat., ce., f., mp., r.

192. Paspalum distichum L. - R. Cerros T. et al. 2593, 2698 (HUMO, MEXU); A.S. Hitchcock 6844 (US). Nat., ce., f., mp.

193. Paspalum hartwegianum E. Fourn. - R. Cerros T. et al. 2613 (HUMO, MEXU); N. Espino V. 14 (MEXU); A.S. Hitchcock 6879 (US); J.A. León 9 (MEXU); E. Simon P. s.n. (MEXU); F.O. Zuloaga et al. 7372 (SI). Nat., r., s.

194. Paspalum humboldtianum Flüggé - A. Brito 178 (MEXU); R. Cerros T. et al. 2594 (HUMO, MEXU); G. Gándara s.n. (MEXU); R.A. Hernández C. et al. 151 (HUMO, UAMIZ); A.S. Hitchcock 6837 (US); E.W.D. Holway 3510 (US); E. Lyonnet 1438 (US), 2529 (MEXU, MO, UAMIZ), 2568 (UAMIZ), 2868 (MEXU, MO, US), s.n. (MEXU); E. Lyonnet \& J. Elcoro 1835 (MEXU, US); A. Miranda S. \& P. Guerrero 350 (MEXU); F. Miranda 3790 (MEXU); J. Morales O. 188 (MEXU); J.N. Rose et al. 10203 (MEXU, US); G. Woronow 2412 (US). Nat., r., s.

195. Paspalum intermedium Munro ex Morong \& Britton - E. Estrada L. 1430 (UAMIZ); G. Gándara s.n. (US). Nat., r., s.

196. Paspalum lentiginosum J. Presl - A.S. Hitchcock 6874 (US); E.W.D. Holway 3514 (US). Nat., s.

197. Paspalum longicuspe Nash - S. Block M. 27 (MEXU); J. Vázquez S. 1742 (MEXU). Nat., r., s.

198. Paspalum melanospermum Desv. ex Poir. - R.A. Hernández C. et al. 578 (HUMO, UAMIZ). Int., $r$. 
Apéndice 2. Continuación.

199. Paspalum notatum Flüggé - E. Estrada L. 1381 (MEXU, UAMIZ), 1724 (MEXU); W. Fosberg 81 (MEXU); R. García M. s.n. (MEXU); B. Guerrero C. 1440 (MEXU, XAL); A.S. Hitchcock 6838 (US); O. López A. 1, 6, 85, 98 (MEXU); E. Lyonnet 971 (MEXU, US), 2432 (MEXU), 2665 (MEXU, MO, US); C.J. Lyons Jr. 149 (MEXU, US); D. Martínez A. 315 (MEXU); A. Miranda S. \& P. Guerrero 343, 376 (MEXU); J. Morales \& O. López 25 (MEXU); M. Núñez M. 125 (MEXU); Salas et al. s.n. (MEXU); C.H. Thomas 48 (MEXU). Nat., ce., f., mp., o. Cult.

200. Paspalum paniculatum L. - R. Cerros T. et al. 2687 (HUMO, MEXU); A. Miranda S. \& P. Guerrero 344, 367 (MEXU); J. Vallejo 7 (UAMIZ). Nat., s.

201. Paspalum plicatulum Michx. - J.T. Camp 48 (MEXU); N. Espino V. 19 (MEXU); A.S. Hitchcock 6854, 6882.5 (US). Nat., f., mp., r.

202. Paspalum pubiflorum Rupr. ex E. Fourn. - R. Guzmán M. 4304, 4318 (IBUG); V. Vázquez T. 294 (XAL); F.O. Zuloaga et al. 7370 (US). Nat., r., s.

203. Paspalum pygmaeum Hack. - R. Cerros T. et al. 2708 (HUMO, MEXU); E. Matuda 25584 (US). Nat., r., s.

204. Paspalum squamulatum E. Fourn. - S. Block M. 119 (MEXU); R. Cerros T. 2540 (HUMO, MEXU); O. López A. 13, 28, 79 (MEXU); E. Lyonnet 2434 (IBUG, MEXU, MO, US); R. Martínez 71 (MEXU); E. Matuda 25919 (MEXU, MO, US); A. Miranda S. \& P. Guerrero 400, 401 (MEXU); J. Morales O. \& López 59 (MEXU); J. Rosasco 126 (MEXU). Nat., op., r., s.

205. Paspalum tenellum Willd. - R. Cerros T. et al. 2581 (HUMO, MEXU); A.S. Hitchcock 938 (MO, US), 6883, s.n. (US); O. López A. 13 a (MEXU); J. Morales O. \& López 58 (MEXU); M. Núñez M. 121 (MEXU); J.R. Reeder \& C.G. Reeder 2212 (MEXU). Nat., op., r.

206. Paspalum tinctum Chase - A.S. Hitchcock 6882 (US). Nat., op., r.

207. Paspalum unispicatum (Scribn. \& Merr.) Nash - F. Amacende A. s.n. (MEXU); L. Pacheco \& P. Dávila A. 62 (MEXU); Y.M.D.O. 12 (HUMO, MEXU). Nat., op., r.

208. Paspalum variabile (E. Fourn.) Nash - A.S. Hitchcock 6833 (US). Nat., op., r.

209. Paspalum virgatum Walter - O. López \& J. Morales O. 34 (MEXU). Nat., op., r.

210. Rottboellia cochinchinensis (Lour.) Clayton - R. Cerros T. et al. 2656 (HUMO, MEXU). Int., mn.

211. Saccharum officinarum L. - J. Vázquez S. 276, 3103 (MEXU). Int., a., r. Cult.

212. Schizachyrium brevifolium (Sw.) Nees ex Büse - R. Cerros T. et al. 2604, 2630, 2699 (HUMO, MEXU); C.G. Pringle 11210 (MEXU, MO, US); J. Sánchez B. PVIIA (MEXU); G. Woronow \& S. Juzepczuk 800 (US). Nat., r., s.

213. Schizachyrium cirratum (Hack.) Wooton \& Standl. - R. Cerros T. et al. 2575 (HUMO, MEXU), 2590 (HUMO); E. Lyonnet 2445 (MEXU, MO, US). Nat., r., s.

214. Schizachyrium microstachyum (Desv. ex Ham.) Roseng. - F.W. Gould 10381 (US); E. Lyonnet 1834 (MEXU, US). Nat., r., s.

215. Schizachyrium sanguineum (Retz.) Alston - R. Cerros T. et al. 2526, 2713 (HUMO, MEXU); R.A. Hernández C. et al. 155 (HUMO, UAMIZ); A.S. Hitchcock 6845 (US); E. Lyonnet 2531 (US); L. Morales s.n. (MEXU). Nat., r., s. 
Apéndice 2. Continuación.

216. Schizachyrium semitectum (Swallen) Reeder - R. Cerros T. et al. 2640 (HUMO, MEXU); G. Itié s.n. (MEXU); J.R. Reeder \& C.G. Reeder 4148 (US). Nat., r., s.

217. Setaria adhaerens (Forssk.) Chiov. - W. Colin C. s.n. (UAMX); A. Quiñones R. s.n. (UAMX). Nat., r., m.

218. Setaria geminata (Forssk.) Veldkamp - M.A. Mijangos C. 6, 59 (MEXU); A. Miranda S. \& P. Guerrero 391 (MEXU); M. Núñez M. s.n. (MEXU). Nat., f., mp.

219. Setaria grisebachii E. Fourn. - C.G. Pringle s.n. (US). Nat., o., r., s.

220. Setaria liebmannii E. Fourn. - R. Cerros T. \& A. Ramírez 1156 (MEXU); F.W. Gould 9576 (US); A. Miranda S. 1186, 1192 (MEXU). Nat., op., r., s.

221. Setaria macrostachya Kunth - E. Estrada L. 1405 (HUMO); A. Huchin G. s.n. (UAMX); A. Rosas H. s.n. (UAMX). Nat., a., f., r., s. Cult.

222. Setaria parviflora (Poir.) Kerguélen - M.C. Avella M. E85 (MEXU); C.E. Boyd 43 (MEXU); J.T. Camp 47 (MEXU); R. Cerros T. 124 (UAMIZ), s.n. (HUMO); R. Cerros T. et al. 2557 (HUMO, MEXU); R.A. Hernández C. et al. 530 (HUMO, UAMIZ); A.S. Hitchcock 6832 (US); A.W. Jackson 65 (MEXU); E. Lyonnet 2415, 2556 (MEXU); $R$. Medina 51 (UAMIZ), s.n. (HUMO); J. Morales \& O. López 57 (MEXU); M. Morales T. 666 (MEXU); C.R. Orcutt 3861 (US); L. Pacheco \& P. Dávila A. 61 (MEXU); C.G. Pringle 7172, 7173, 9587 (US); A. Pulido E. et al. 22, 43 (HUMO); M. Reyes B. 1174 (MEXU); A. Sotelo G. 293 (MEXU); J. Vázquez S. 2633, 3429 (MEXU). Nat., mp., r.

223. Setaria pumila (Poir.) Roem. \& Schult. - R. Cerros T. 1599 (HUMO); J.T. Harris 69 (US). Int., f., r. Cult.

224. Setariopsis auriculata (E. Fourn.) Scribn. - G. Andrade \& C.H. Ramos s.n. (MEXU); M.L. Aranda s.n. (MEXU); R. Cerros T. 2500 a (HUMO, MEXU); R. Cerros T. et al. 2534 (HUMO, MEXU); F. Gallegos H. 669 (MEXU); A. Miranda S. 1193, 1208 (MEXU); J. Morales O. 91, 134 (MEXU); C.R. Orcutt 4428 (US); V. Peñaloza G. 819 (MEXU); M. Reyes B. 1298 (MEXU); J.N. Rose et al. 8538 (US); C.M. Rowell \& G.L. Webster 2274 (MEXU). Nat., r., s.

225. Setariopsis latiglumis (Vasey) Scribn. - R. Cerros T. et al. 2559 (HUMO, MEXU); E. Lyonnet 2642 (MEXU); A. Miranda S. \& P. Guerrero 362 (MEXU); J. Morales O. 141 (MEXU); C.G. Pringle 11246 (MEXU, MO, US); A. Sotelo G. 102 (MEXU). Nat., r., s.

226. Sorghastrum incompletum (J. Presl) Nash - E. Castrejón V. 1395 (HUMO); R. Cerros T. et al. 2694 (HUMO, MEXU); G. Gándara s.n. (US); R. García \& C. Díaz P. s.n. (UAMX); R. González R. \& C. Díaz P. s.n. (UAMX); E. Lima \& C. Díaz P. s.n. (UAMX); E. Lyonnet \& J. Elcoro 1837 (US); A. Miranda S. \& M. Serralde s.n. (MEXU); J. Sánchez B. PXIVA (MEXU); M.E. Vela G. \& C. Díaz P. s.n. (UAMX). Nat., r., s.

227. Sorghastrum nudipes Nash - E. Lyonnet 631 (MO). Nat., r., s.

228. Sorghastrum nutans (L.) Nash - E. Lyonnet 1838 a (MEXU). Nat., r., s.

229. Sorghastrum stipoides (Kunth) Nash - O. López A. 88 (MEXU). Nat., r., s.

230. Sorghum bicolor (L.) Moench - M.F. Alanis A. \& C. Díaz P. s.n. (UAMX); A. Cabrera D. \& C. Díaz P. s.n. (UAMX); A. Campechano T. \& C. Díaz P. s.n. (UAMX); A. 
Apéndice 2. Continuación.

Castañeda C. \& C. Díaz P. s.n. (UAMX); F.O.L. et al. s.n. (UAMX); A. Garcés G. \& C. Díaz P. s.n. (UAMX); A. García A. \& C. Díaz P. s.n. (UAMX); M.A. Herrera S. \& C. Díaz P. s.n. (UAMX); A. Ibarra \& C. Díaz P. s.n. (UAMX); D. Luna S. et al. s.n. (UAMX); P. Miranda M. \& C. Díaz P. s.n. (UAMX); K. Monroy H. \& C. Díaz P. s.n. (UAMX); H. Olvera A. \& C. Díaz P. s.n. (UAMX); P. Patixtán G. \& C. Díaz P. s.n. (UAMX); M. Pucheta D. \& C. Díaz P. s.n. (UAMX); M. Quezada R. 1826 (HUMO); G. Quintero L. \& C. Díaz P. s.n. (UAMX); M. Reyes J. \& C. Díaz P. s.n. (UAMX); Y. Richard \& C. Díaz P. s.n. (UAMX); J. Rojas A. \& C. Díaz P. s.n. (UAMX); H. Rufino \& C. Díaz P. s.n. (UAMX); V.Q.P.L. \& C. Díaz P. s.n. (UAMX); F.U. Vidal L. \& C. Díaz P. s.n. (UAMX); I. Zamora \& C. Díaz P. s.n. (UAMX). Int., c., f., r. Cult.

231. Sorghum halepense (L.) Pers. - V. Anario R. \& C. Díaz P. s.n. (UAMX); G. Barrera C. \& C. Díaz P. s.n. (UAMX); F. Bautista et al. s.n. (UAMX); S. Casado A. \& C. Díaz P. s.n. (UAMX); E.M. Castillo R. et al. s.n. (UAMX); I. Castro L. et al. s.n. (UAMX); R. Cerros T. et al. 2596 (HUMO, MEXU); J.A. Chávez A. \& C. Díaz P. s.n. (UAMX); M.A.D. Díaz A. et al. s.n. (UAMX); L.G. Espinosa R. \& C. Díaz P. s.n. (UAMX); M.L. Gallardo R. et al. s.n. (UAMX); R. García \& C. Díaz P. s.n. (UAMX); J.M. García A. \& C. Díaz P. s.n. (UAMX); M. García G. \& C. Díaz P. s.n. (UAMX); S. Gaytán O. et al. s.n. (UAMX); J.L. Gómez C. \& C. Díaz P. s.n. (UAMX); M. Gutiérrez P. s.n. (HUMO); G. Hernández M. \& C. Díaz P. s.n. (UAMX); Y.E. López J. \& C. Díaz P. s.n. (UAMX); M. Luna A. \& C. Díaz P. s.n. (UAMX); J.L. Marín V. \& C. Díaz P. s.n. (UAMX); J. Martineau J. et al. s.n. (UAMX); V. Méndez R. \& C. Díaz P. s.n. (UAMX); R.I. Pineda A. \& C. Díaz P. s.n. (UAMX); M.A. Rodríguez R. et al. s.n. (UAMX); F. Romero P. \& C. Díaz P. s.n. (UAMX); J. Rzedowski 35615 (MO); V. Sánchez M. \& C. Díaz P. s.n. (UAMX); J.L. Sánchez P. s.n. (MEXU). Intr., mn.

232. Steinchisma cuprea (Hitchc. \& Chase) W.V. Br. - M.S. Antipovitch 73 (MO); R. Cerros T. et al. 2600 (HUMO, MEXU); J. Sánchez B. PXXA, PXX1A (MEXU). Nat., r., s.

233. Stenotaphrum secundatum (Walter) Kuntze - B. Flores M. \& C. Díaz P. s.n. (UAMX); R. Granados A. \& C. Díaz P. s.n. (UAMX); A. Miranda S. \& P. Guerrero 368 (MEXU); B. Osorio R. \& C. Díaz P. s.n. (UAMX); J. Vázquez S. 3931 (MEXU). Int., ce., f., mp., o. Cult.

234. Themeda quadrivalvis (L.) Kuntze - R. Cerros T. et al. 2675 (HUMO, MEXU); J.G. Sánchez-Ken et al. s.n. (IBUG, MEXU, XAL). Int., m.

235. Trachypogon spicatus (L.) Kuntze - R. Cerros T. et al. 2576, 2621 (HUMO, MEXU); L. Crispín F. \& C. Díaz P. s.n. (UAMX); M.S. González A. s.n. (UAMX); R.A. Hernández C. et al. 32, 536 (HUMO, UAMIZ); M. Luna A. \& C. Díaz P. s.n. (UAMX); J.L. Marín V. \& C. Díaz P. s.n. (UAMX); J. Morales O. 190 (MEXU); L. Peña O. \& C. Díaz P. s.n. (UAMX); E. Pineda G. \& C. Díaz P. s.n. (UAMX); C.G. Pringle 6514 (US); P.G. Russell \& M.J. Souviron 261 (US); J. Sánchez B. PXIXA (MEXU); Y. Santiago G. \& C. Díaz P. s.n. (UAMX); G. Woronow \& S. Juzepczuk 950 (US). Nat., r., s.

236. Tripsacum dactyloides (L.) L. - R. Cerros T. 2511 (HUMO, MEXU); R. Cerros T. et al. 2718 (HUMO, MEXU); R.A. Hernández C. et al. 532 (HUMO, UAMIZ); D. Martínez A. s.n. (MEXU); L.F. Randolph \& F.R. Randolph s.n. (US). Nat., m., f., r. Cult. 
Apéndice 2. Continuación.

237. Tripsacum lanceolatum Rupr. ex. E. Fourn. - R. Cerros T. 1597 (HUMO); A.S. Hitchcock 6840 (US); E. Lyonnet 2421, 2645 (US), 2552 (MEXU, US); Seler 4348 (US). Nat., m., f., r. Cult.

238. Tripsacum latifolium Hitchc. - A.J. Sharp 441355 (US). Nat., m., f., r. Cult.

239. Tripsacum pilosum Scribn. \& Merr. - E. Lyonnet 2654 (US). Nat., m., f., r. Cult.

240. Urochloa brizantha (Hochst. ex A. Rich.) R.D. Webster - V.V. Vega Q. s.n. (UAMX). Int., f., m., r. Cult.

241. Urochloa discifera (E. Fourn.) Morrone \& Zuloaga - R. Cerros T. 2515 (HUMO, MEXU); F. Ramírez H. s.n. (UAMX). Nat., s., r.

242. Urochloa fusca (Sw.) B.F. Hansen \& Wunderlin - M.A. Ávila 6 (MEXU); A. Miranda S. 1203 (MEXU); A. Miranda S. \& P. Guerrero 393 (MEXU); M. Reyes B. 1307 (MEXU, UAMIZ); sin colector s.n. (MEXU); J. Vázquez S. 1786 (MEXU). Nat., f., op., r.

243. Urochloa meziana (Hitchc.) Morrone \& Zuloaga - G. Gándara 32 (US). Nat., r., s.

244. Urochloa mollis (Sw.) Morrone \& Zuloaga - M. Almazán V. s.n. (UAMX); J. Galicia X. s.n. (UAMX); A. Huchin G. s.n. (UAMX). Nat., r.

245. Urochloa mutica (Forssk.) T.Q. Nguyen - M. Núñez M. 88 (MEXU). Int., ce., f., r. Cult.

246. Urochloa panicoides P. Beauv. - M.C. Ávalos E. et al. s.n. (UAMX); W. Celis C. et al. s.n. (UAMX); R. Cerros T. 2501 (HUMO, MEXU); R. Cerros T. et al. 2550, 2646 (HUMO, MEXU); H.J. Cortés B. et al. s.n. (UAMX); N.O. Fernández M. \& R.L.M. Velasco S. s.n. (UAMX); C.A. Fuentes T. s.n. (UAMX); T. García M. \& A.M. Prieto S. s.n. (UAMX); F. García et al. s.n. (UAMX); I. González A. et al. s.n. (UAMX); S. Luna G. s.n. (UAMX); E. Martínez A. \& A.G. Roa L. s.n. (UAMX); A. Miranda S. 1183, 1212, 1217 (MEXU); J. Morales O. 112 (MEXU); E. Moreno P. et al. s.n. (UAMX); A.M. Prieto S. s.n. (UAMX); A.E. Reséndiz C. et al. s.n. (UAMX); H. Saldívar E. s.n. (UAMX); M. Saldivar E. \& C. Díaz P. s.n. (UAMX). Int., mn., r.

247. Urochloa plantaginea (Link) R.D. Webster - M.C. Celso M. s.n. (UAMX); R. Cerros T. et al. 2532, 2553, 2602 (HUMO, MEXU); N. Espino V. 11, 16 (MEXU); G. Gándara 28 (MEXU); A.S. Hitchcock 6853 (US); O. López A. 32, 34, 94 (MEXU); E. Lyonnet 2530 (MEXU, US), 2662 (MEXU); A. Miranda S. 1222 (MEXU); A. Miranda S. \& P. Guerrero 341 (MEXU); L. Morales s.n. (MEXU); C.R. Orcutt 3890 (MO, US); M. Reyes B. 1137, 1139 (MEXU, UAMIZ), 1316 (MEXU); E. Tovar s.n. (UAMX). Int., r.

248. Urochloa reptans (L.) Stapf - A. Miranda S. 1197, 1213, 1218 (MEXU); G. Velázquez O. s.n. (UAMX). Int., f., r. Cult.

249a. Zea mays L. subsp. mays - F. Díaz \& G. Laris s.n. (UAMX); Equipo 6 \& C. Díaz P. s.n. (UAMX); E.A. Jacinto M. \& C. Díaz P. s.n. (UAMX); D. Luna S. et al. s.n. (UAMX); W.V. Rodin 17 (MEXU). Nat., c., f., r. Cult.

249b. Zea mays L. subsp. parviglumis Iltis \& Doebley - G. Gándara 21 (US). Nat., f., mp., r. Cult.

250. Zeugites pringlei Scribn. - G. Barroso C. et al. 15 (UAMIZ); E. Lyonnet 972 (MEXU, MO, US); E. Lyonnet \& J. Elcoro 1803 (MEXU, MO, US); C.G. Pringle 7174, 8361 (MEXU, MO, US), 11250 (F, MO, US, WIS), 13896 (F, MICH, MO, US). Nat., op., s. 
Apéndice 2. Continuación.

251. Zeugites smilacifolius Scribn. - W. Boege 1974 (MO); R.A. Hernández C. et al. 93, 612 (HUMO, UAMIZ); I.K. Langman 3688 (US); C.G. Pringle 5961 (MO, US), 11251 (MO, US, WIS), 6604 (BM, MO, US). Nat., op., s.

252. Zuloagaea bulbosa (Kunth) Bess - S. Block M. 1145 (MEXU). Nat., f., op., r.

Pooideae Benth.

253. Achnatherum constrictum (Hitchc.) Valdés-Reyna \& Barkworth - R.A. Hernández C. et al. 593 (HUMO, UAMIZ). Nat., s.

254. Agrostis bourgeaei E. Fourn. - E. Lyonnet 2518 (MEXU, UAMIZ, US). Nat., s.

255. Agrostis ghiesbreghtii E. Fourn. - E. Matuda 26005 (MEXU); J. Rzedowski 21622 (MO). Nat., s.

256. Agrostis perennans (Walter) Tuck. - L. Cardoso V. 349 (MEXU); E. Matuda 25591 (MEXU, MO); J. Morales O. 169 (MEXU); J. Vázquez S. 3904 (MEXU). Nat., r., s.

257. Agrostis schaffneri E. Fourn. - Fassett 28438 (MEXU); P.R. Mastosic et al. 1274 (MEXU). Nat., r., s.

258. Agrostis tolucensis Kunth - T.J. Amezcua et al. 60 (MEXU); F. Gallegos H. 660 (US); E. Lyonnet 2497 (MEXU, MO, US); E. Matuda s.n. (MEXU). Nat., r., s.

259. Anthoxanthum mexicanum (Rupr. ex. E. Fourn.) Mez - E. Matuda 25594 (MEXU, US). Nat., s.

260. Avena fatua L. - J. Espinosa 41 (MEXU); A.G. José 286 (MEXU, UAMIZ); A. Pulido E. 54 (MEXU, UAMIZ); M. Reyes B. 1172 (MEXU). Int., me., mp., r., f. Cult.

261. Avena sativa L. - G. Barroso C. et al. 19 (MEXU); I. Díaz V.\& R. Noriega T. 1116, 1123 (MEXU); J. Espinosa 57 (MEXU); M. Flores et al. 116 (MEXU, UAMIZ); J.J. Ortiz 1130 (MEXU, MO); V. Sánchez et al. 21 (MEXU, UAMIZ); A. Sotelo G. 270 (MEXU). Int., me., mp., r., f. Cult.

262. Brachypodium latifolium E. Fourn. - G. Ayala A. 25 (IEB, MEXU), 39 (MEXU); S. Block M. 1040 (MEXU). Nat., s.

263. Brachypodium mexicanum (Roem. \& Schult.) Link - G. Ayala A. 12, 68 (MEXU); S. Block M. 1200 (MEXU); W. Celis C. s.n. (UAMX); R. Cerros T. 2544 (HUMO, MEXU); E. Lyonnet 2509 (MO, UAMIZ, US), 2522 (MEXU, US); J. Morales O. 160 (MEXU); L. Morales P. s.n. (UAMX); J.R. Reeder 3043 (MEXU); sin colector s.n. (MEXU). Nat., s.

264. Briza minor L. - G. Ayala A. 105 (MEXU); J.G. Azael H. s.n. (UAMX); S. Block M. 395 (MEXU); R. Cerros T. et al. 2545, 2664 (HUMO, MEXU); Q.F. Fuchs 1931 (MEXU); A. González \& B. Rodríguez s.n. (MEXU); J. Morales O. 153 (MEXU); L. Morales P. s.n. (UAMX); M. Núñez M. 75 (MEXU); J.J. Ortiz 1129 (MEXU); P. Tenorio L. 18971 (MEXU). Int., o., r. Cult.

265. Bromus anomalus Rupr. ex E. Fourn. - G. Andrade s.n. (MEXU); G.L. Fisher 120 (US); E. Lyonnet 47 (MO), 2495 (MEXU, MO, US); G.B. van Schaack 3418 (MICH). Nat., f., s.

266. Bromus carinatus Hook. \& Arn. - G. Ayala A. 51 (MEXU); S. Block M. 448 (MEXU); P. Fernández s.n. (MEXU); A.S. Hitchcock 6885 (US); O. Martínez R. s.n. (UAMX); 
Apéndice 2. Continuación.

A. Miranda S. 406 (MEXU); J.J. Ortiz 1134, 1135 (MEXU, MO); A. Pulido E. et al. 40, 103 (UAMIZ); A. Sotelo G. 352 (MEXU). Nat., ce., f., o.

267. Bromus catharticus Vahl - M.E. Espino N. s.n. (UAMX); H.D. Terrones D. s.n. (UAMX). Int., r.

268. Bromus diandrus Roth - R. Cerros T. et al. 2666, 2714 (HUMO, MEXU); A. Huchin G. \& J. Galicia J. s.n. (UAMX); E. Oviedo 75 (MEXU); J.A. Montaño 4 (MEXU); J. Rosado 106 (MEXU). Int., r. Cult.

269. Bromus dolichocarpus Wagnon - S. Block M. 1047, 1051, 1184, 1214 (MEXU); R. Cerros T. et al. 2704 (HUMO); E. Lyonnet \& J. Elcoro 1811 (MEXU, US), 1812 (MEXU); E. Manrique et al. 733 (MEXU). Nat., s.

270. Calamagrostis orizabae Steud. - L. Cardoso V. 1542 (UAMIZ). Nat., s.

271. Calamagrostis tolucensis (Kunth) Trin. ex Steud. - M. Gutiérrez P. 645 (MEXU); G.B. Ross s.n. (US). Nat., s.

272. Chascolytrum subaristatum (Lam.) Desv. - C.A. Lázaro S. s.n. (UAMX); A. Soberanes s.n. (UAMX). Nat., r., s.

273. Cinna poiformis (Kunth) Scribn. \& Merr. - M.A. Bernal Y. s.n. (UAMX); E. Lyonnet 2520 (MEXU, US). Nat., r., s.

274. Dactylis glomerata L. - E. Oviedo 76 (MO). Int., f., mn., o., r. Cult.

275. Deschampsia elongata (Hook.) Munro ex Benth. - E. Lyonnet 2459 (MEXU, US); J. Morales O. 163 (MEXU). Nat., r., s.

276. Elymus elymoides (Raf.) Swezey - G. Ayala A. 52 (MEXU). Nat., ce., f., r. Cult.

277. Festuca amplissima Rupr. - R. Cerros T. et al. 2705 (HUMO, MEXU); E. Lyonnet 2482 (MEXU, MO, US). Nat., r., s.

278. Festuca breviglumis Swallen - E. Lyonnet \& J. Elcoro 1812 (US). Nat., r., s.

279. Festuca lugens (E. Fourn.) Hitchc. ex Hern.-Xol. - I. Escamilla G. 20, 23, 27 (MEXU); G.L. Fisher s.n. (MO); N. Lyons 129 (MEXU); J. Morales O. 150, 157 (MEXU); M. Núñez M. 86 (MEXU); C.R. Orcutt 3748 (MEXU). Nat., r., s.

280. Festuca orizabensis E.B. Alexeev - L. Cardoso V. 21 (HUMO). Nat., r., s.

281. Festuca rosei Piper - E. Lyonnet 2471 (MEXU); E. Matuda 25612 (MEXU); C.R. Orcutt 3743 (MEXU). Nat., r., s.

282. Festuca tolucensis Kunth - H.H. Iltis et al. 109 (MEXU). Nat., r., s.

283. Glyceria striata (Lam.) Hitchc. - J. Bonilla 1485 (MEXU, MO); J. Bonilla 1508, 1517, 1537, 1561 (MEXU, MO), 1524 (MEXU, MO, UAMIZ); L. Cardoso V. 1246 (MEXU); E. Lyonnet \& L. Ch ves 3401 (US); R.F. McAdams 44 (MEXU). Nat., r., s.

284. Hordeum jubatum L. - C.I. Mamani O. s.n. (UAMX); C. Ortega A. s.n. (UAMX). Nat., m., $r$.

285. Hordeum vulgare L.- W. Fosberg 24 (MEXU); V. Jaramillo V. et al. 924 (MEXU); J. Vázquez S. 3958 (MEXU). Int., c., f., m., r. Cult.

286. Jarava ichu Ruiz \& Pav. - G. Ayala A. 46, 61, 85 (MEXU); L. Cardoso V. 1464 (HUMO); I. Escamilla G. 19 (MEXU); G. Flores C. \& R. Monroy M. 7597 (MEXU); G. Gándara 26 (MEXU); E. Lyonnet 57 (MEXU); P.R. Mastosic \& L. Cardoso V. 
Apéndice 2. Continuación.

1273 (MEXU); M. Núñez M. 42 (MEXU); M. Reyes B. 1038 (MEXU); sin colector 71 (MEXU). Nat., ce., op., r., s.

287. Lolium multiflorum Lam. - J.M. González Z. s.n. (UAMX); F. Morales J. s.n. (UAMX); I. Navarro s.n. (UAMX); E. Oviedo 77 (MO). Int., ce., f., mp., op. Cult.

288. Lolium perenne L. - I. Díaz V. \& R. Noriega T. 1126 (HUMO); G.B. Ross s.n. (US). Int., ce., f., mp., op. Cult.

289. Nassella mucronata (Kunth) R.W. Pohl - G. Ayala A. 50 (MEXU); R. Martínez 88 (MEXU). Nat., r., s.

290. Peyritschia deyeuxioides (Kunth) Finot - G. Ayala A. 101 (MEXU); J. Espinosa 53 (MEXU); M. Núñez M. 72 (MEXU); J.J. Ortiz 1131 (MEXU, MO); C.G. Pringle 13984 (MO, US); J. Vázquez S. 3923 (MEXU). Nat., op., r.

291. Peyritschia koelerioides (Peyr.) E. Fourn. - E. Lyonnet 2499 (MEXU, US); J. Morales O. $151 a$ (MEXU); C.R. Orcutt 3740 (MEXU); J.R. Reeder \& C.G. Reeder 3045 (ARIZ, US). Nat., op., r.

292. Phalaris canariensis L. - M. Gutiérrez P. 444 (MEXU). Int., a., f., m., r. Cult.

293. Piptochaetium fimbriatum (Kunth) Hitchc. - L.L. Garza G. s.n. (MEXU). Nat., op., r.

294. Piptochaetium seleri (Pilg.) Henr. - E.G. Carney 59 (MEXU); G.L. Fisher 108 (US); E. Lyonnet 2511 (MEXU), 2512 (MEXU, MO); J.D. Traylor 61 (MEXU). Nat., op., r.

295. Piptochaetium virescens (Kunth) Parodi - S. Block M. 198, 447 (MEXU); J. Castañeda R. 1001 (MEXU); R. Cerros T. 2542 (HUMO, MEXU); R. Cerros T. et al. 2702, 2703 (HUMO, MEXU); E. Lyonnet 2498 (US); E. Lyonnet \& J. Elcoro 1826 (US); J.J. Ortiz 1132 (MEXU, MO). Nat., op., r.

296. Poa annua L. - R. Cerros T. et al. 2548, 2707 (HUMO, MEXU); A. Contreras R. \& C. Díaz P. s.n. (UAMX); V. Escalante \& C. Díaz P. s.n. (UAMX); A. García M. \& C. Díaz P. s.n. (UAMX); S. Gaytán O. \& C. Díaz P. s.n. (UAMX); M.E. López V. \& C. Díaz P. s.n. (UAMX); M.S. Macías L. \& C. Díaz P. s.n. (UAMX); F. Martínez M. \& C. Díaz P. s.n. (UAMX); O. Martínez R. \& C. Díaz P. s.n. (UAMX); T. Mondragón L. \& C. Díaz P. s.n. (UAMX); N.M. Morales A. \& C. Díaz P. s.n. (UAMX); C.R. Orcutt 3858 (US); G. Pérez S. \& C. Díaz P. s.n. (UAMX); A. Pulido E. et al. 60, 63 (UAMIZ); R. Salazar \& C. Díaz P. s.n. (UAMX); F. Terán H. \& C. Díaz P. s.n. (UAMX); I. Valencia M. \& C. Díaz P. s.n. (UAMX); J.V. Verref H. \& C. Díaz P. s.n. (UAMX). Int., mn., o., r. Cult.

297. Polypogon elongatus Kunth - A.S. Hitchcock 6848 (US). Nat., r., s.

298. Polypogon interruptus Kunth - R. Barranco V. 9 (UAMIZ); E. Lyonnet 2430 (HUMO). Nat., r., s.

299. Polypogon viridis (Gouan) Breistr. - C.G. Pringle 7160 (US); J. Vázquez S. 3831 (MEXU). Int., r.

300. Schedonorus arundinaceus (Schreb.) Dumort. - G. Ayala A. 74 (MEXU); R. Vallejo 9 (HUMO). Int., ce., f., o. Cult.

301. Triniochloa micrantha (Scribn.) Hitchc. - C.G. Pringle 8018, 9172, 11214 (MO, US). Nat., r., s. 
Apéndice 2. Continuación.

302. Triniochloa stipoides (Kunth) Hitchc. - R. Beutelspacher C. \& C. Díaz P. s.n. (UAMX); I.M. Cano C. \& C. Díaz P. s.n. (UAMX); Y. Carbajal R. et al. s.n. (UAMX); I. Castro M. \& C. Díaz P. s.n. (UAMX); R.A. Hernández C. et al. 244, 616 (HUMO, UAMIZ); R. Hernández L. \& C. Díaz P. s.n. (UAMX); E. Lyonnet 630, 1535 (MO, US); E. Lyonnet \& J. Elcoro 1773 (US); C.G. Pringle 11754, 15001 (US); O. Vázquez Z. \& C. Díaz P. s.n. (UAMX). Nat., r., s.

303. Trisetum irazuense (Kuntze) Hitchc. - E. Lyonnet 2503 (MEXU, US). Nat., r., s.

304. Trisetum virletii E. Fourn. - T.J. Amezcua et al. 25 (HUMO), 26 (MEXU); G. Ayala A. 7, 71 (MEXU); R.A. Hernández C. et al. 257 (HUMO, UAMIZ); G.B. Hinton 17531bis (HUMO); R.M. King \& R. Johnson Jr. 4155 (US); E. Lyonnet 3210 (MEXU, MO); E. Manrique et al. 737 (MEXU); E. Matuda 1215 (US); C.G. Pringle 11758, 15002 (US); A. Pulido E. et al. 27, 37 (HUMO); J. Vázquez S. 2354 (MEXU). Nat., r., s.

305. Triticum aestivum L. - I. Díaz V. \& R. Noriega T. 1120 (MEXU). Int., c., f., m., r. Cult.

306. Vulpia myuros (L.) C.C. Gmel. - N.A. Angeles G. \& C. Díaz P. s.n. (UAMX); S. Block M. 407 (MEXU); E. Camacho D. \& C. Díaz P. s.n. (UAMX); L. Cardoso V. 1350 (HUMO); R. Cerros T. et al. 2547, 2658, 2662, 2710 (HUMO, MEXU); C.J. de Anda B. \& C. Díaz P. s.n. (UAMX); C.A. Fuentes T. \& C. Díaz P. s.n. (UAMX); J. Galicia X. \& C. Díaz P. s.n. (UAMX); J. García C. 190 (MEXU, MO); E. Lyonnet 2439 (US); T. Mondragón L. \& C. Díaz P. s.n. (UAMX); K. Monroy H. \& C. Díaz P. s.n. (UAMX); C.R. Orcutt 3860 (MO, US); J.J. Ortiz 1136 (MO); F. Robles P. \& C. Díaz P. s.n. (UAMX); R. Salazar \& C. Díaz P. s.n. (UAMX); A. Soberanes s.n. (UAMX); P. Tenorio L. 18978 (MEXU). Int., ce., mp., r. Cult. 
